\title{
REEB GRAPHS OF CURVES ARE STABLE UNDER FUNCTION PERTURBATIONS
}

\author{
B. DI FABIO AND C. LANDI
}

\begin{abstract}
Reeb graphs provide a method to combinatorially describe the shape of a manifold endowed with a Morse function. One question deserving attention is whether Reeb graphs are robust against function perturbations. Focusing on 1-dimensional manifolds, we define an editing distance between Reeb graphs of curves, in terms of the cost necessary to transform one graph into another through editing moves. Our main result is that changes in Morse functions induce smaller changes in the editing distance between Reeb graphs of curves, implying stability of Reeb graphs under function perturbations. We also prove that our editing distance is equal to the natural pseudo-distance, and, moreover, that it is lower bounded by the bottleneck distance of persistent homology.
\end{abstract}

\section{INTRODUCTION}

The shape similarity problem has been studied since long by the computer vision community for dealing with shape classification and retrieval tasks. Comparison of 2D images is often dealt with considering just the silhouette or contour curve of the studied object. Shape properties, such as curvature, are encoded in compact representations of shapes, namely, shape descriptors. In this framework, shape similarity can be measured by defining an appropriate distance on the set of the chosen shape descriptors.

A question that deserves attention is the choice of the distance used to compare shape descriptors. Indeed, it is clear that any data acquisition is subject to perturbations, noise and approximation errors and, without stability, distinct computational investigations of the same object could produce completely different results. So a major problem in shape comparison concerns the stability against data perturbations.

In this paper we focus on the Reeb graph shape descriptor for curves. Reeb graphs have been used as an effective tool for shape analysis and description tasks since [17, 18], in the case of surfaces, even if they were introduced in [15] as a topological construct for manifolds of any dimension.

Reeb graphs of curves endowed with simple Morse functions are simply cycle graphs with an even number of vertices corresponding alternatively to the maxima and minima of the function. We also equip vertices of Reeb graphs with the value taken by the function at the corresponding critical points.

Our main contribution is the construction of a combinatorial distance between Reeb graphs of curves such that changes in functions imply smaller changes in the distance:

MAIN RESUlT (Theorem 4.6). For two simple Morse functions $f, g: S^{1} \rightarrow \mathbb{R}$, the editing distance between the associated labeled Reeb graphs is never greater than $\|f-g\|_{C^{0}}$.

2010 Mathematics Subject Classification. Primary 05C10, 68T10; Secondary 54C30.

Key words and phrases. shape similarity, editing distance, Morse function, natural stratification, natural pseudo-distance. 
This proves the stability of Reeb graphs of curves under perturbations.

Our distance is based on an adaptation of the well-known notion of editing distance between graphs [19]. We introduce three basic types of editing operations, represented in Table 1, corresponding to the insertion (birth) of a new pair of adjacent points of maximum and minimum, the deletion (death) of such a pair, and the relabeling of the vertices. A cost is associated with each of these operations. Then our distance is given by the infimum of the costs necessary to transform a graph into another by using these editing operations.

The main idea of the proof is to consider the linear path $\lambda g+(1-\lambda) f$ between $f$ and $g$ in the space of smooth real functions on $S^{1}$ and the corresponding one-parameter family of Reeb graphs. Assuming genericity, the changes that the functions undergo along the linear path can be translated into editing operations (insertions, deletions and relabelings) on the corresponding Reeb graphs. Some care must be taken in order to reduce to a situation in which genericity of the path can be assumed. By appropriately taking a discretization of the path, we show that each editing operation has a cost that is not greater than the $C^{0}$-norm evaluated at the difference between the corresponding functions. In particular, this requires a stability result for critical values of Morse functions.

As a further contribution of this paper, our editing distance is compared to other distances that can be used to measure shape similarity of curves: the natural pseudo-distance [8] and the bottleneck distance [5]. These distances share the stability under function perturbations property. We prove that our editing distance coincides with the natural pseudodistance, thus obtaining a tool for its study. Moreover, we prove that the bottleneck distance is never greater than the editing distance and we exhibit an example in which it is strictly smaller. Hence, the bottleneck distance does not discriminate shapes as thoroughly as the editing distance.

The paper is organized as follows. After recalling the basic properties of labeled Reeb graphs of closed curves in Section 1, in Section 2 we give the definition of the admissible deformations transforming a Reeb graph into another, the cost associated with each kind of deformation, and the definition of an editing distance in terms of this cost. Section 3 is mainly devoted to prove that our editing distance is actually a metric. In Section 4 we firstly show that our distance is locally stable, that is each simple Morse function $f$ has a neighborhood consisting of functions $g$ such that for $f$ and $g$ the main result holds. Then, we show that our distance is globally stable, that is the main result holds for any two simple Morse functions $f, g$. We end the paper by comparing the editing distance with the natural pseudo-distance and the bottleneck distance in Section 5. For the reader's convenience, the result about the stability of critical values of Morse functions (for a manifold of any dimension), obtained through homological arguments, is given in the Appendix.

\section{LABELED REEB GRAPHS OF CLOSED CURVES}

Throughout the paper, $\mathscr{F}$ denotes the set of $C^{\infty}$ real functions on $S^{1}$. For $f \in \mathscr{F}$, we denote by $K(f)$ the set of its critical points. If $p \in K(f)$, then the real number $f(p)$ is called a critical value of $f$, and the set $\left\{q \in S^{1}: q \in f^{-1}(f(p))\right\}$ is called a critical level of $f$. Otherwise, if $p \in S^{1} \backslash K(f)$, then $f(p)$ is called a regular value. Moreover, a critical point $p$ is called non-degenerate if and only if the second derivative of $f$ at $p$ is non-zero.

A function $f \in \mathscr{F}$ is called a Morse function if all its critical points are non-degenerate. Besides, a Morse function is said to be simple if each critical level contains exactly one critical point. The set of simple Morse functions will be denoted by $\mathscr{F}^{0}$, as a reminder that it is a sub-manifold of $\mathscr{F}$ of co-dimension 0 (see also Section 4). 
Let $f \in \mathscr{F}^{0}$. The Reeb graph $\Gamma_{f}$ associated with $f$ is a cycle graph on an even number of vertices, corresponding, alternatively, to the minima and maxima of $f$ on $S^{1}$ [14].

The vertex set of $\Gamma_{f}$ will be denoted by $V\left(\Gamma_{f}\right)$, and its edge set by $E\left(\Gamma_{f}\right)$. Moreover, if $v_{1}, v_{2} \in V\left(\Gamma_{f}\right)$ are adjacent vertices, i.e., connected by an edge, we will write $e\left(v_{1}, v_{2}\right) \in$ $E\left(\Gamma_{f}\right)$.

We label the vertices of $\Gamma_{f}$, by equipping each of them with the value of $f$ at the corresponding critical point. We denote such a labeled graph by $\left(\Gamma_{f}, f_{\mid}\right)$, where $f_{\mid}: V\left(\Gamma_{f}\right) \rightarrow \mathbb{R}$ is the restriction of $f: S^{1} \rightarrow \mathbb{R}$ to $K(f)$. A simple example is displayed in Figure $1(a)-(c)$.

To facilitate the reader, in all the figures of this paper we shall adopt the convention of representing $f$ as the height function, so that $f_{l}\left(v_{a}\right)<f_{l}\left(v_{b}\right)$ if and only if $v_{a}$ is lower than $v_{b}$ in the picture. Moreover, we will often identify each $v \in V\left(\Gamma_{f}\right)$ with the corresponding $p \in K(f)$.

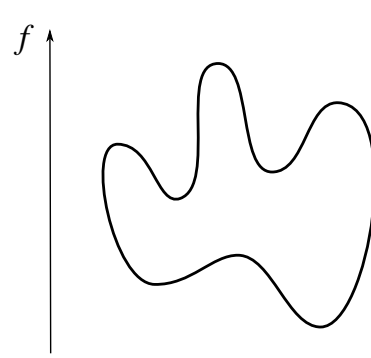

(a)

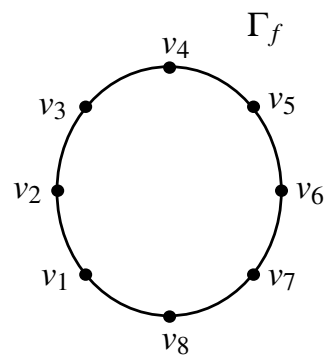

(b)

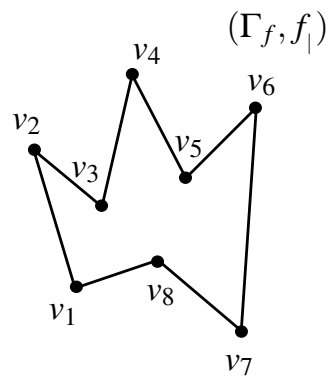

(c)

FIGURE 1. (a) $f: S^{1} \rightarrow \mathbb{R}$ is the height function; (b) the associated Reeb graph $\Gamma_{f} ;(c)$ the associated labeled Reeb graph $\left(\Gamma_{f}, f_{\mid}\right)$. Here labels are represented by the heights of the vertices.

Definition 1.1. Two functions $f, g \in \mathscr{F}^{0}$ are called topologically equivalent if there exists a diffeomorphism $\xi: S^{1} \rightarrow S^{1}$ and an orientation preserving diffeomorphism $\eta: \mathbb{R} \rightarrow \mathbb{R}$ such that $g(\xi(p))=\eta(f(p))$ for every $p \in S^{1}$.

Given two topologically equivalent functions $f, g \in \mathscr{F}^{0}$, it is well-known that the associated Reeb graphs, $\Gamma_{f}$ and $\Gamma_{g}$, are isomorphic graphs, i.e., there exists an edge-preserving bijection $\Phi: V\left(\Gamma_{f}\right) \rightarrow V\left(\Gamma_{g}\right)$. Beyond that, an even stronger result holds. Two functions $f, g \in \mathscr{F}^{0}$ are topologically equivalent if and only if such a bijection $\Phi$ also preserves the vertices order, i.e., for every $v, w \in V\left(\Gamma_{f}\right), f(v)<f(w)$ if and only if $g(\Phi(v))<g(\Phi(w))$. The preceding result has been used by Arnold in [1] to classify simple Morse functions up to the topological equivalence relation.

The natural definition of isomorphism between labeled Reeb graphs is the following one.

Definition 1.2. We shall say that two labeled Reeb graphs $\left(\Gamma_{f}, f_{\mid}\right),\left(\Gamma_{g}, g_{\mid}\right)$are isomorphic if there exists an edge-preserving bijection $\Phi: V\left(\Gamma_{f}\right) \rightarrow V\left(\Gamma_{g}\right)$ such that $f_{\mid}=g_{\mid} \circ \Phi$.

The following Proposition 1.5 provides a necessary and sufficient condition in order that two labeled Reeb graphs are isomorphic. It is based on the next definition of reparameterization equivalent functions.

Definition 1.3. Let $\mathscr{H}\left(S^{1}\right)$ be the set of homeomorphisms on $S^{1}$. We shall say that two functions $f, g \in \mathscr{F}^{0}$ are re-parameterization equivalent if there exists $\tau \in \mathscr{H}\left(S^{1}\right)$ such that $f=g \circ \tau$. 
Lemma 1.4. Let $\left(\Gamma_{f}, f_{\mid}\right)$and $\left(\Gamma_{g}, g_{\mid}\right)$be labeled Reeb graphs. If an edge-preserving bijection $\Phi: V\left(\Gamma_{f}\right) \rightarrow V\left(\Gamma_{g}\right)$ exists, then there also exists $\tau \in \mathscr{H}\left(S^{1}\right)$ such that $\tau_{\left.\right|_{V\left(\Gamma_{f}\right)}}=\Phi$. If moreover $f_{\mid}=g_{\mid} \circ \Phi$, then $f=g \circ \tau$.

Proof. The proof of the first statement is inspired by [8, Lemma 4.2]. Let us construct $\tau$ by extending $\Phi$ to $S^{1}$ as follows. Let us recall that $V\left(\Gamma_{f}\right)=K(f)$ and $V\left(\Gamma_{g}\right)=K(g)$, and, by abuse of notation, for every pair of adjacent vertices $p^{\prime}, p^{\prime \prime} \in V\left(\Gamma_{f}\right)$, let us identify the edge $e\left(p^{\prime}, p^{\prime \prime}\right) \in E\left(\Gamma_{f}\right)$ with the arc of $S^{1}$ having endpoints $p^{\prime}$ and $p^{\prime \prime}$, and not containing any other critical point of $f$. For every $p \in K(f)$, let $\tau(p)=\Phi(p)$. Now, let us define $\tau(p)$ for every $p \in S^{1} \backslash K(f)$. Given $p \in S^{1} \backslash K(f)$, we observe that there always exist $p^{\prime}, p^{\prime \prime} \in V\left(\Gamma_{f}\right)$ such that $p \in e\left(p^{\prime}, p^{\prime \prime}\right)$. Since $\Phi$ is edge-preserving, there exists $e\left(\Phi\left(p^{\prime}\right), \Phi\left(p^{\prime \prime}\right)\right)=e\left(\tau\left(p^{\prime}\right), \tau\left(p^{\prime \prime}\right)\right) \in E\left(\Gamma_{g}\right)$. Hence, we can define $\tau(p)$ as the unique point of $e\left(\tau\left(p^{\prime}\right), \tau\left(p^{\prime \prime}\right)\right)$ such that, if $f(p)=\left(1-\lambda_{p}\right) f\left(p^{\prime}\right)+\lambda_{p} f\left(p^{\prime \prime}\right)$, with $\lambda_{p} \in[0,1]$, then $g(\tau(p))=\left(1-\lambda_{p}\right) g\left(\tau\left(p^{\prime}\right)\right)+\lambda_{p} g\left(\tau\left(p^{\prime \prime}\right)\right)$. Clearly, $\tau$ belongs to $\mathscr{H}\left(S^{1}\right)$.

As for the second statement, it is sufficient to observe that, if $f_{\mid}=g \circ \Phi$, since $\tau(p)=$ $\Phi(p)$ for every $p \in K(f)$, then clearly $f_{\mid}(p)=g_{\mid}(\tau(p))$ for every $p \in K(f)$. Moreover, for every $p \in S^{1} \backslash K(f)$, by the construction of $\tau$, it holds that $g(\tau(p))=\left(1-\lambda_{p}\right) g\left(\Phi\left(p^{\prime}\right)\right)+$ $\lambda_{p} g\left(\Phi\left(p^{\prime \prime}\right)\right)=\left(1-\lambda_{p}\right) f\left(p^{\prime}\right)+\lambda_{p} f\left(p^{\prime \prime}\right)=f(p)$. In conclusion, $f(p)=g(\tau(p))$ for every $p \in S^{1}$, and, hence, $f, g$ are re-parameterization equivalent.

Proposition 1.5 (Uniqueness theorem). Let $\left(\Gamma_{f}, f_{\mid}\right),\left(\Gamma_{g}, g_{\mid}\right)$be labeled Reeb graphs. Then $\left(\Gamma_{f}, f_{\mid}\right)$is isomorphic to $\left(\Gamma_{g}, g_{\mid}\right)$if and only if $f$ and $g$ are re-parameterization equivalent.

Proof. The direct statement is a trivial consequence of Lemma 1.4.

As for the converse statement, it is sufficient to observe that any $\tau \in \mathscr{H}\left(S^{1}\right)$ such that $f=g \circ \tau$, as well as its inverse $\tau^{-1}$, takes the minima of $f$ to the minima of $g$ and the maxima of $f$ to the maxima of $g$. Hence, $\Phi: V\left(\Gamma_{f}\right) \rightarrow V\left(\Gamma_{g}\right)$, with $\Phi=\tau_{\left.\right|_{V\left(\Gamma_{f}\right)}}$, is an edge-preserving bijection such that $f_{\mid}=g_{\mid} \circ \Phi$.

As a consequence of Proposition 1.5, two labeled Reeb graphs isomorphic in the sense of Definition 1.2 will always be identified, and in such case we will simply write $\left(\Gamma_{f}, f_{\mid}\right)=$ $\left(\Gamma_{g}, g_{\mid}\right)$.

The following Proposition 1.6 ensures that, for every cycle graph on an even number of vertices, appropriately labeled, there exists a unique (up to re-parameterization) $f \in \mathscr{F}^{0}$, having such a graph as the associated labeled Reeb graph.

Proposition 1.6 (Realization theorem). Let $(G, \ell)$ be a labeled graph, where $G$ is a cycle graph on an even number of vertices, and $\ell: V(G) \rightarrow \mathbb{R}$ is an injective function such that, for any vertex $v_{2}$ adjacent (that is connected by an edge) to the vertices $v_{1}$ and $v_{3}$, either both $\ell\left(v_{1}\right)$ and $\ell\left(v_{3}\right)$ are smaller than $\ell\left(v_{2}\right)$, or both $\ell\left(v_{1}\right)$ and $\ell\left(v_{3}\right)$ are greater than $\ell\left(v_{2}\right)$. Then there exists a simple Morse function $f: S^{1} \rightarrow \mathbb{R}$ such that $\left(\Gamma_{f}, f_{\mid}\right)=(G, \ell)$.

Proof. It is evident.

\section{Editing DisTANCE BETWEEN LABELED REEB GRAPHS}

We now define the editing deformations admissible to transform a labeled Reeb graph of a closed curve into another. We introduce at first elementary deformations and then the deformations obtained by their composition. Next, we associate a cost with each type of deformation, and define a distance between labeled Reeb graphs in terms of such a cost. 


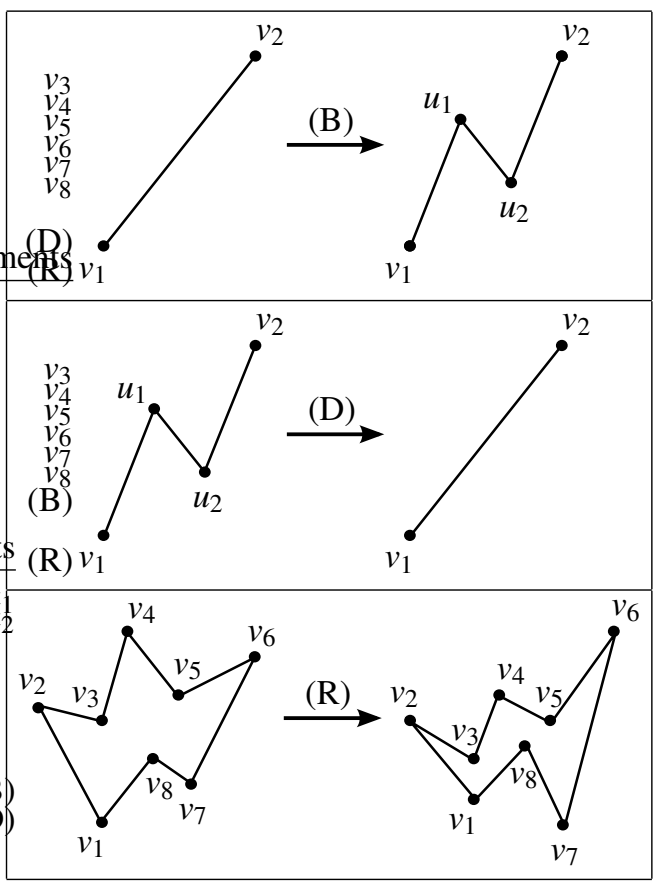

TABLE 1. The upper two figures schematically show the elementary deformations of type (B) and (D), respectively; the third figure shows an example of elementary deformation of type (R).

Definition 2.1. Let $\left(\Gamma_{f}, f_{l}\right)$ be a labeled Reeb graph with $2 n$ vertices, $n \geq 1$. We call $T$ an elementary deformation of $\left(\Gamma_{f}, f_{t}\right)$ if $T$ transforms $\left(\Gamma_{f}, f_{t}\right)$ in one and only one of the following ways:

(B) (Birth): Fix an edge $e\left(v_{1}, v_{2}\right) \in E\left(\Gamma_{f}\right)$, with $f_{l}\left(v_{1}\right)<f_{l}\left(v_{2}\right)$. Then $T$ transforms $\left(\Gamma_{f}, f_{\mid}\right)$into a labeled graph $(G, \ell)$ according to the following rule: $G$ is the new graph on $2 n+2$ vertices, obtained deleting the edge $e\left(v_{1}, v_{2}\right)$ and inserting two new vertices $u_{1}, u_{2}$ and the edges $e\left(v_{1}, u_{1}\right), e\left(u_{1}, u_{2}\right), e\left(u_{2}, v_{2}\right)$; moreover, $\ell: V(G) \rightarrow \mathbb{R}$ is defined by extending $f$ from $V\left(\Gamma_{f}\right)$ to $V(G)=V\left(\Gamma_{f}\right) \cup\left\{u_{1}, u_{2}\right\}$ in such a way that $\ell_{\mid V\left(\Gamma_{f}\right)} \equiv f_{\mid}$, and $f_{\mid}\left(v_{1}\right)<\ell\left(u_{2}\right)<\ell\left(u_{1}\right)<f_{\mid}\left(v_{2}\right)$.

(D) (Death): Assume $n \geq 2$, and fix edges $e\left(v_{1}, u_{1}\right), e\left(u_{1}, u_{2}\right), e\left(u_{2}, v_{2}\right) \in E\left(\Gamma_{f}\right)$, with $f_{\mid}\left(v_{1}\right)<f_{\mid}\left(u_{2}\right)<f_{\mid}\left(u_{1}\right)<f_{\mid}\left(v_{2}\right)$. Then $T$ transforms $\left(\Gamma_{f}, f_{\mid}\right)$into a labeled graph $(G, \ell)$ according to the following rule: $G$ is the new graph on $2 n-2$ vertices, obtained deleting $u_{1}, u_{2}$ and the edges $e\left(v_{1}, u_{1}\right), e\left(u_{1}, u_{2}\right), e\left(u_{2}, v_{2}\right)$, and inserting an edge $e\left(v_{1}, v_{2}\right)$; moreover, $\ell: V(G) \rightarrow \mathbb{R}$ is defined as the restriction of $f$ to $V\left(\Gamma_{f}\right) \backslash\left\{u_{1}, u_{2}\right\}$.

(R) (Relabeling): $T$ transforms $\left(\Gamma_{f}, f_{1}\right)$ into a labeled graph $(G, \ell)$ according to the following rule: $G=\Gamma_{f}$, and for any vertex $v_{2}$ adjacent to the vertices $v_{1}$ and $v_{3}$ (possibly $v_{1} \equiv v_{3}$ for $n=1$ ), if both $f_{\mid}\left(v_{1}\right)$ and $f_{1}\left(v_{3}\right)$ are smaller (greater, respectively) than $f_{\mid}\left(v_{2}\right)$, then both $\ell\left(v_{1}\right)$ and $\ell\left(v_{3}\right)$ are smaller (greater, respectively) than $\ell\left(v_{2}\right)$; moreover, for every $v \neq w, \ell(v) \neq \ell(w)$. 
We shall denote by $T\left(\Gamma_{f}, f_{\mid}\right)$the result of the elementary deformation $T$ applied to $\left(\Gamma_{f}, f_{\mid}\right)$.

Table 1 schematically illustrates the elementary deformations described in Definition 2.1.

Proposition 2.2. Let $T$ be an elementary deformation of $\left(\Gamma_{f}, f_{\mid}\right)$, and let $(G, \ell)=T\left(\Gamma_{f}, f_{\mid}\right)$. Then $(G, \ell)$ is a labeled Reeb graph $\left(\Gamma_{g}, g_{\mid}\right)$, and $g \in \mathscr{F}^{0}$ is unique up to re-parameterization equivalence.

Proof. The claim follows from Propositions 1.6 and 1.5.

As a consequence of the above result, from now on, we will directly write $T\left(\Gamma_{f}, f_{\mid}\right)=$ $\left(\Gamma_{g}, g_{\mid}\right)$. Moreover, we can also apply elementary deformations iteratively. This fact is used in the next Definition 2.3.

Given an elementary deformation $T$ of $\left(\Gamma_{f}, f_{\mid}\right)$and an elementary deformation $S$ of $T\left(\Gamma_{f}, f_{\mid}\right)$, the juxtaposition $S T$ means applying first $T$ and then $S$.

Definition 2.3. We shall call deformation of $\left(\Gamma_{f}, f_{\mid}\right)$any finite ordered sequence $T=$ $\left(T_{1}, T_{2}, \ldots, T_{r}\right)$ of elementary deformations such that $T_{1}$ is an elementary deformation of $\left(\Gamma_{f}, f_{\mid}\right), T_{2}$ is an elementary deformation of $T_{1}\left(\Gamma_{f}, f_{\mid}\right), \ldots, T_{r}$ is an elementary deformation of $T_{r-1} T_{r-2} \cdots T_{1}\left(\Gamma_{f}, f_{\mid}\right)$. We shall denote by $T\left(\Gamma_{f}, f_{\mid}\right)$the result of the deformation $T$ applied to $\left(\Gamma_{f}, f_{l}\right)$.

Let us define the cost of a deformation.

Definition 2.4. Let $T$ be an elementary deformation transforming $\left(\Gamma_{f}, f_{\mid}\right)$into $\left(\Gamma_{g}, g_{\mid}\right)$.

- If $T$ is of type (B) inserting the vertices $u_{1}, u_{2} \in V\left(\Gamma_{g}\right)$, then we define the associated cost as

$$
c(T)=\frac{\left|g_{\mid}\left(u_{1}\right)-g_{\mid}\left(u_{2}\right)\right|}{2} ;
$$

- If $T$ is of type (D) deleting the vertices $u_{1}, u_{2} \in V\left(\Gamma_{f}\right)$, then we define the associated cost as

$$
c(T)=\frac{\left|f_{\mid}\left(u_{1}\right)-f_{\mid}\left(u_{2}\right)\right|}{2} ;
$$

- If $T$ is of type (R) relabeling the vertices $v \in V\left(\Gamma_{f}\right)=V\left(\Gamma_{g}\right)$, then we define the associated cost as

$$
c(T)=\max _{v \in V\left(\Gamma_{f}\right)}\left|f_{\mid}(v)-g_{\mid}(v)\right| .
$$

Moreover, if $T=\left(T_{1}, \ldots, T_{r}\right)$ is a deformation such that $T_{r} \cdots T_{1}\left(\Gamma_{f}, f_{\mid}\right)=\left(\Gamma_{g}, g_{\mid}\right)$, we define the associated cost as $c(T)=\sum_{i=1}^{r} c\left(T_{i}\right)$.

We now introduce the concept of inverse deformation.

Definition 2.5. Let $T$ be a deformation such that $T\left(\Gamma_{f}, f_{\mid}\right)=\left(\Gamma_{g}, g_{\mid}\right)$. Then we denote by $T^{-1}$, and call it the inverse of $T$, the deformation such that $T^{-1}\left(\Gamma_{g}, g_{\mid}\right)=\left(\Gamma_{f}, f_{\mid}\right)$defined as follows:

- If $T$ is elementary of type (B) inserting two vertices, then $T^{-1}$ is of type (D) deleting the same vertices;

- If $T$ is elementary of type (D) deleting two vertices, then $T^{-1}$ is of type (B) inserting the same vertices, with the same labels; 
- If $T$ is elementary of type (R) relabeling vertices of $V\left(\Gamma_{f}\right)$, then $T^{-1}$ is again of type $(\mathrm{R})$ relabeling these vertices in the inverse way;

- If $T=\left(T_{1}, \ldots, T_{r}\right)$, then $T^{-1}=\left(T_{r}^{-1}, \ldots, T_{1}^{-1}\right)$.

Proposition 2.6. For every deformation $T$ such that $T\left(\Gamma_{f}, f_{\mid}\right)=\left(\Gamma_{g}, g_{\mid}\right), c\left(T^{-1}\right)=c(T)$.

Proof. Trivial.

We prove that, for every two labeled Reeb graphs, a finite number of elementary deformations always allows us to transform any of them into the other one. We recall that we identify labeled Reeb graphs that are isomorphic according to Definition 1.2. We first need a lemma, stating that in any labeled Reeb graph with at least four vertices we can find two adjacent vertices that can be deleted.

Lemma 2.7. Let $\left(\Gamma_{f}, f_{\mid}\right)$be a labeled Reeb graph with at least four vertices. Then there exist $e\left(v_{1}, u_{1}\right), e\left(u_{1}, u_{2}\right), e\left(u_{2}, v_{2}\right) \in E\left(\Gamma_{f}\right)$, with $f_{\mid}\left(v_{1}\right)<f_{\mid}\left(u_{2}\right)<f_{\mid}\left(u_{1}\right)<f_{\mid}\left(v_{2}\right)$.

Proof. It is sufficient to take adjacent vertices $u_{1}$ and $u_{2}$ such that $f_{\mid}\left(u_{1}\right)-f_{\mid}\left(u_{2}\right)$ is equal to $\min _{e(u, v) \in E\left(\Gamma_{f}\right)}\left|f_{1}(u)-f_{\mid}(v)\right|$.

Proposition 2.8. Let $\left(\Gamma_{f}, f_{\mid}\right)$and $\left(\Gamma_{g}, g_{\mid}\right)$be two labeled Reeb graphs. Then the set of all the deformations $T$ such that $T\left(\Gamma_{f}, f_{1}\right)=\left(\Gamma_{g}, g_{\mid}\right)$is non-empty. This set of deformations will be denoted by $\mathscr{T}\left(\left(\Gamma_{f}, f_{\mid}\right),\left(\Gamma_{g}, g_{\mid}\right)\right)$.

Proof. Let $m$ and $n$ be the number of vertices of $\Gamma_{f}$ and $\Gamma_{g}$, respectively.

If $m=n$, then it is sufficient to take an elementary deformation $T$ of type (R) to transform $\left(\Gamma_{f}, f_{1}\right)$ into $\left(\Gamma_{g}, g_{1}\right)$. Otherwise, let us suppose $m>n$. Then $m \geq 4$ and, by Lemma 2.7, we can apply a finite sequence of elementary deformations of type (D) to $\left(\Gamma_{f}, f_{\mid}\right)$, so that in the resulting labeled Reeb graph $\left(\Gamma_{h}, h_{\mid}\right), \Gamma_{h}$ has only $n$ vertices. Now, $\left(\Gamma_{h}, h_{\mid}\right)$can be transformed into $\left(\Gamma_{g}, g_{\mid}\right)$through an elementary deformation of type (R). For the case $m<n$, the same proof applies with deformations of type (B) instead of deformations of type (D).

A simple example explaining the above proof is given in Figure 2. We point out that

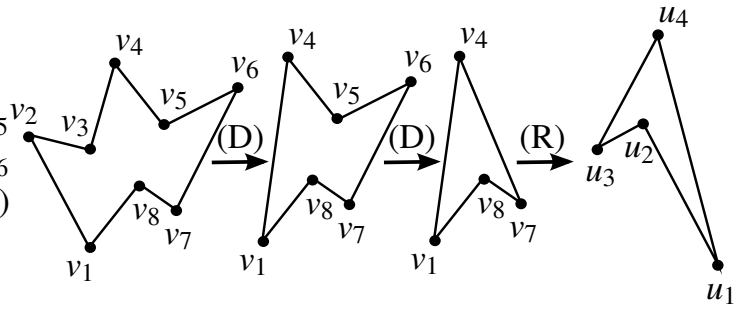

FIGURE 2. The leftmost labeled Reeb graph is transformed into the rightmost one applying first two elementary deformations of type (D), then one elementary deformation of type (R).

the deformation constructed in the proof of Proposition 2.8 is not necessarily the cheapest one, as can be seen in Example 2.

We now introduce an editing distance between labeled Reeb graphs, in terms of the cost necessary to transform one graph into another. 
Theorem 2.9. For every two labeled Reeb graphs $\left(\Gamma_{f}, f_{\mid}\right)$and $\left(\Gamma_{g}, g_{\mid}\right)$, we set

$$
d\left(\left(\Gamma_{f}, f_{\mid}\right),\left(\Gamma_{g}, g_{\mid}\right)\right)=\inf _{T \in \mathscr{T}\left(\left(\Gamma_{f}, f_{\mid}\right),\left(\Gamma_{g}, g_{\mid}\right)\right)} c(T) .
$$

Then $d$ is a distance.

The proof of the above theorem will be postponed to the end of the following section. Indeed, even if the properties of symmetry and triangular inequality can be easily verified, the property of the positive definiteness of $d$ is not straightforward because the set of all possible deformations transforming $\left(\Gamma_{f}, f_{\mid}\right)$into $\left(\Gamma_{g}, g_{\mid}\right)$is not finite. In order to prove the positive definiteness of $d$, we will need a further result concerning the connection between the editing distance between two labeled Reeb graphs, $\left(\Gamma_{f}, f_{\mid}\right),\left(\Gamma_{g}, g_{\mid}\right)$, and the natural pseudo-distance between the associated functions $f, g$.

\section{A LOWER BOUND FOR THE EDITING DISTANCE}

The natural pseudo-distance is a measure of the dissimilarity between two continuous functions $\varphi: X \rightarrow \mathbb{R}, \psi: Y \rightarrow \mathbb{R}$, with $X$ and $Y$ compact, homeomorphic topological spaces. Roughly speaking, it is defined as the infimum of the variation of the values of $\varphi$ and $\psi$, when we move from $X$ to $Y$ through homeomorphisms (see $[6,7,8]$ for more details).

The following Theorem 3.1 states that the natural pseudo-distance computed between the simple Morse functions $f: S^{1} \rightarrow \mathbb{R}$ and $g: S^{1} \rightarrow \mathbb{R}$ is a lower bound for the editing distance between the associated labeled Reeb graphs.

Such a lower bound is useful for achieving two different results: firstly, the proof of Theorem 2.9, i.e., that $d$ is a distance (see Corollary 3.2); secondly, the computation, in certain simple cases, of the value of $d$ (see, e.g., Examples 1-2).

In Section 5 we will show that the editing distance is actually equal to the natural pseudo-distance.

Theorem 3.1. Let $f, g \in \mathscr{F}^{0}$, and $\left(\Gamma_{f}, f_{\mid}\right),\left(\Gamma_{g}, g_{\mid}\right)$the associated labeled Reeb graphs. Then $d\left(\left(\Gamma_{f}, f_{\mid}\right),\left(\Gamma_{g}, g_{\mid}\right)\right) \geq \inf _{\tau \in \mathscr{H}\left(S^{1}\right)}\|f-g \circ \tau\|_{C^{0}}$.

Proof. Let us prove that, for every $T \in \mathscr{T}\left(\left(\Gamma_{f}, f_{\mid}\right),\left(\Gamma_{g}, g_{l}\right)\right), c(T) \geq \inf _{\tau \in \mathscr{H}\left(S^{1}\right)}\|f-g \circ \tau\|_{C^{0}}$.

First of all, assume that $T$ is an elementary deformation transforming $\left(\Gamma_{f}, f_{l}\right)$ into $\left(\Gamma_{g}, g_{\mid}\right)$. For conciseness, slightly abusing notations, we will identify arcs of $S^{1}$ having as endpoints two critical points $p^{\prime}, p^{\prime \prime} \in V\left(\Gamma_{f}\right)$, and not containing other critical points of $f$, with the edges $e\left(p^{\prime}, p^{\prime \prime}\right) \in E\left(\Gamma_{f}\right)$.

(1) Let $T$ be of type (R) relabeling vertices of $V\left(\Gamma_{f}\right)$. Since, by Definition $2.1(\mathrm{R})$, $\Gamma_{f}=\Gamma_{g}$, we can always apply Lemma 1.4 , considering $\Phi$ as the identity map, to obtain a homeomorphism $\tau$ on $S^{1}$ such that $\tau(p)=p$ for every $p \in K(f)$. As far as non-critical points are concerned, following the proof of Lemma 1.4, for every $p \in S^{1} \backslash K(f), \tau(p)$ is defined as that point on $S^{1}$ such that, if $p \in e\left(p^{\prime}, p^{\prime \prime}\right) \in$ $E\left(\Gamma_{f}\right)$, with $f(p)=\left(1-\lambda_{p}\right) f\left(p^{\prime}\right)+\lambda_{p} f\left(p^{\prime \prime}\right), \lambda_{p} \in[0,1]$, then $\tau(p) \in e\left(p^{\prime}, p^{\prime \prime}\right)$ with $g(\tau(p))=\left(1-\lambda_{p}\right) g\left(p^{\prime}\right)+\lambda_{p} g\left(p^{\prime \prime}\right)$. Therefore, by substituting to $f(p)$ and $g(\tau(p))$ the above expressions, we see that $\max _{p \in S^{1}}|f(p)-g(\tau(p))|=\max _{p \in V\left(\Gamma_{f}\right)} \mid f_{1}(p)-$ $g_{\mid}(p) \mid=c(T)$.

(2) Let $T$ be of type (D) deleting $q_{1}, q_{2} \in V\left(\Gamma_{f}\right)$, the edges $e\left(p_{1}, q_{1}\right), e\left(q_{1}, q_{2}\right), e\left(q_{2}, p_{2}\right)$, and inserting the edge $e\left(p_{1}, p_{2}\right)$. Thus, for every $p \in K(f) \backslash\left\{q_{1}, q_{2}\right\}, f(p)=g(p)$. 
It is not restrictive to assume that $f\left(p_{1}\right)<f\left(q_{2}\right)<f\left(q_{1}\right)<f\left(p_{2}\right)$. Then we can define a sequence $\left(\tau_{n}\right)$ of homeomorphisms on $S^{1}$ approximating this elementary deformation. Let $\tau_{n}(p)=p$ for every $p \in V\left(\Gamma_{f}\right) \backslash\left\{q_{1}, q_{2}\right\}=V\left(\Gamma_{g}\right)$ and $n \in \mathbb{N}$. Moreover, let $\bar{q}$ be the point of $e\left(p_{1}, p_{2}\right) \in E\left(\Gamma_{g}\right)$ such that $g(\bar{q})=\frac{f\left(q_{1}\right)+f\left(q_{2}\right)}{2}$ (such a point $\bar{q}$ exists because $g\left(p_{1}\right)=f\left(p_{1}\right)<f\left(q_{2}\right)<f\left(q_{1}\right)<f\left(p_{2}\right)=g\left(p_{2}\right)$ and it is unique because we are assuming that no critical points of $g$ occur in the considered arc). Let us fix a positive real number $c<\min \left\{g\left(p_{2}\right)-g(\bar{q}), g(\bar{q})-g\left(p_{1}\right)\right\}$. For every $n \in \mathbb{N}$, let us define $\tau_{n}\left(q_{1}\right)$ (resp. $\tau_{n}\left(q_{2}\right)$ ) as the only point on $S^{1}$ belonging to the arc with endpoints $p_{1}, \bar{q}$ (resp. $\left.\bar{q}, p_{2}\right)$ contained in $e\left(p_{1}, p_{2}\right)$, such that $g\left(\tau_{n}\left(q_{1}\right)\right)=g(\bar{q})-\frac{c}{n}\left(\right.$ resp. $\left.g\left(\tau_{n}\left(q_{2}\right)\right)=g(\bar{q})+\frac{c}{n}\right)$ as shown in Figure 3. Now, let us linearly extend $\tau_{n}$ to all $S^{1}$ in the following way. For every $p \in S^{1} \backslash K(f)$, if $p$ belongs to the arc with endpoints $p^{\prime}, p^{\prime \prime} \in K(f)$ not containing any other critical point, and is such that $f(p)=\left(1-\lambda_{p}\right) f\left(p^{\prime}\right)+\lambda_{p} f\left(p^{\prime \prime}\right), \lambda_{p} \in[0,1]$, then $\tau_{n}(p)$ belongs to the arc with endpoints $\tau_{n}\left(p^{\prime}\right), \tau_{n}\left(p^{\prime \prime}\right)$ not containing any other critical point, and is such that $g\left(\tau_{n}(p)\right)=\left(1-\lambda_{p}\right) g\left(\tau_{n}\left(p^{\prime}\right)\right)+\lambda_{p} g\left(\tau_{n}\left(p^{\prime \prime}\right)\right)$. Hence, $\tau_{n}$ is a homeomorphism on $S^{1}$ for every $n \in \mathbb{N}$, and $\lim _{n \rightarrow \infty} \max \left|f(p)-g\left(\tau_{n}(p)\right)\right|=$ $\lim _{n \rightarrow \infty} \max _{p \in V\left(\Gamma_{f}\right)}\left|f(p)-g\left(\tau_{n}(p)\right)\right|=\lim _{n \rightarrow \infty} \max \left\{f\left(q_{1}\right)-g\left(\tau_{n}\left(q_{1}\right)\right), f\left(q_{2}\right)-g\left(\tau_{n}\left(q_{2}\right)\right)\right\}=$ $\left|f\left(q_{1}\right)-g(\bar{q})\right|=\frac{f_{l}\left(q_{1}\right)-f_{\mid}\left(q_{2}\right)}{2}=c(T)$.

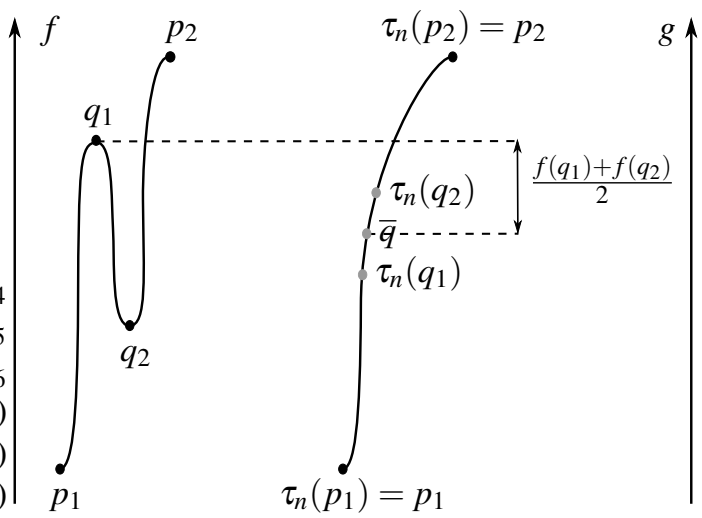

FIGURE 3. The construction of the homomorphism $\tau_{n}$ as described in step (2) of the proof of Theorem 3.1. The arc $e\left(p_{1}, q_{1}\right)\left(e\left(q_{1}, q_{2}\right)\right.$, and $e\left(q_{2}, p_{2}\right)$, respectively) is homeomorphically taken to the arc having $\tau_{n}\left(p_{1}\right), \tau_{n}\left(q_{1}\right)\left(\tau_{n}\left(q_{1}\right), \tau_{n}\left(q_{2}\right)\right.$ and $\tau_{n}\left(q_{2}\right), \tau_{n}\left(p_{2}\right)$, respectively) as endpoints.

(3) Let $T$ be of type (B) deleting $e\left(p_{1}, p_{2}\right) \in E\left(\Gamma_{f}\right)$, and inserting two vertices $q_{1}, q_{2}$ and the edges $e\left(p_{1}, q_{1}\right), e\left(q_{1}, q_{2}\right), e\left(q_{2}, p_{2}\right)$. Then we can apply the same proof as (2), by considering the inverse deformation $T^{-1}$ that, by Definition 2.5, is of type (D) and, by Proposition 2.6, has the same cost of $T$.

Therefore, observing that in (1), the homeomorphism $\tau$ can be clearly replaced by a sequence $\left(\tau_{n}\right)$, with $\tau_{n}=\tau$ for every $n \in \mathbb{N}$, we can assert that, for every elementary deformation $T$, there exists a sequence of homeomorphisms on $S^{1},\left(\tau_{n}\right)$, such that $c(T)=$ $\lim _{n \rightarrow \infty}\left\|f-g \circ \tau_{n}\right\|_{C^{0}} \geq \inf _{\tau \in \mathscr{H}\left(S^{1}\right)}\|f-g \circ \tau\|_{C^{0}}$. 
Now, let $T=\left(T_{1}, \ldots, T_{r}\right) \in \mathscr{T}\left(\left(\Gamma_{f}, f_{\mid}\right),\left(\Gamma_{g}, g_{\mid}\right)\right)$and prove that, also in this case, $c(T) \geq$ $\inf _{\tau \in \mathscr{H}\left(S^{1}\right)}\|f-g \circ \tau\|_{C^{0}}$. Let us set $T_{i} \cdots T_{1}\left(\Gamma_{f}, f_{\mid}\right)=\left(\Gamma_{f^{(i)}}, f_{\mid}^{(i)}\right), f=f^{(0)}, g=f^{(r)}$. For $i=1, \ldots, r$, let $\left(\tau_{n}^{(i)}\right)_{n}$ be a sequence of homeomorphisms on $S^{1}$ for which it holds that $c\left(T_{i}\right)=\lim _{n \rightarrow \infty}\left\|f^{(i-1)}-f^{(i)} \circ \tau_{n}^{(i)}\right\|_{C^{0}}$, and let $\left(\tau_{n}^{(0)}\right)_{n}$ be the constant sequence such that $\tau_{n}^{(0)}$ is the identity map for every $n \in \mathbb{N}$. Then

$$
\begin{aligned}
& c(T)=\sum_{i=1}^{r} c\left(T_{i}\right)=\lim _{n \rightarrow \infty}\left\|f^{(0)}-f^{(1)} \circ \tau_{n}^{(1)}\right\|_{C^{0}}+\sum_{i=1}^{r-1} \lim _{n \rightarrow \infty}\left\|f^{(i)}-f^{(i+1)} \circ \tau_{n}^{(i+1)}\right\|_{C^{0}} \\
& =\lim _{n \rightarrow \infty}\left\|f^{(0)}-f^{(1)} \circ \tau_{n}^{(1)}\right\|_{C^{0}} \\
& +\sum_{i=1}^{r-1} \lim _{n \rightarrow \infty}\left\|f^{(i)} \circ \tau_{n}^{(i)} \circ \ldots \circ \tau_{n}^{(0)}-f^{(i+1)} \circ \tau_{n}^{(i+1)} \circ \tau_{n}^{(i)} \circ \cdots \circ \tau_{n}^{(0)}\right\|_{C^{0}} \\
& \geq \lim _{r \rightarrow \infty}\left\|f^{(0)}-f^{(r)} \circ \tau_{n}^{(r)} \circ \tau_{n}^{(r-1)} \circ \cdots \circ \tau_{n}^{(0)}\right\|_{C^{0}} \geq \inf _{\tau \in \mathscr{H}\left(S^{1}\right)}\|f-g \circ \tau\|_{C^{0}}
\end{aligned}
$$

where the third equality is obtained by observing that

$f^{(i)} \circ \tau_{n}^{(i)} \circ \cdots \circ \tau_{n}^{(0)}-f^{(i+1)} \circ \tau_{n}^{(i+1)} \circ \tau_{n}^{(i)} \circ \cdots \circ \tau_{n}^{(0)}=\left(f^{(i)}-f^{(i+1)} \circ \tau_{n}^{(i+1)}\right) \circ \tau_{n}^{(i)} \circ \cdots \circ \tau_{n}^{(0)}$

for every $i \in\{1, \ldots, r-1\}$, and that $\|\cdot\|_{C^{0}}$ is invariant under re-parameterization; the first inequality is consequent to the triangular inequality.

Corollary 3.2. If $d\left(\left(\Gamma_{f}, f_{\mid}\right),\left(\Gamma_{g}, g_{\mid}\right)\right)=0$ then $\left(\Gamma_{f}, f_{\mid}\right)=\left(\Gamma_{g}, g_{\mid}\right)$.

Proof. From Theorem 3.1, $d\left(\left(\Gamma_{f}, f_{\mid}\right),\left(\Gamma_{g}, g_{\mid}\right)\right)=0$ implies that $\inf _{\tau \in \mathscr{H}\left(S^{1}\right)}\|f-g \circ \tau\|_{C^{0}}=0$. In [3] it has been proved that when $\inf _{\tau \in \mathscr{H}(X, Y)}\|f-g \circ \tau\|_{C^{0}}=0$, with $X, Y$ two closed curves of class at least $C^{2}$, a homeomorphism $\bar{\tau} \in \mathscr{H}(X, Y)$ exists such that $f=g \circ \bar{\tau}$. Therefore, the claim follows from Proposition 1.5.

Proof of Theorem 2.9. The positive definiteness of $d$ has been proved in Corollary 3.2; the symmetry is a consequence of Proposition 2.6; the triangular inequality can be easily verified in the standard way.

Now we describe two simple examples showing how it is possible to compute the editing distance between two labeled Reeb graphs, $\left(\Gamma_{f}, f_{\mid}\right),\left(\Gamma_{g}, g_{\mid}\right)$, by exploiting the knowledge of the natural pseudo-distance value between $f$ and $g$. In particular, Example 1 provides a situation in which the infimum cost over all the deformations transforming $\left(\Gamma_{f}, f_{\mid}\right)$into $\left(\Gamma_{g}, g_{\mid}\right)$is actually a minimum. In Example 2 this infimum is obtained by applying a passage to the limit.

Example 1. Let us consider $f, g: S^{1} \rightarrow \mathbb{R}$, with $f, g \in \mathscr{F}^{0}$, depicted in Figure 4. We now show that $d\left(\left(\Gamma_{f}, f_{\mid}\right),\left(\Gamma_{g}, g_{\mid}\right)\right)=\frac{1}{2}\left(f\left(q_{1}\right)-f\left(p_{1}\right)\right)$. Indeed, in this case, the natural pseudodistance between $f$ and $g$ is equal to $\frac{1}{2}\left(f\left(q_{1}\right)-f\left(p_{1}\right)\right)$ (cf. [8]). Therefore, by Theorem 3.1, it follows that $d\left(\left(\Gamma_{f}, f_{\mid}\right),\left(\Gamma_{g}, g_{\mid}\right)\right) \geq \frac{1}{2}\left(f\left(q_{1}\right)-f\left(p_{1}\right)\right)$. On the other hand, the deformation $T$ of type (D) that deletes the vertices $p_{1}, q_{1} \in V\left(\Gamma_{f}\right)$, the edges $e\left(p, q_{1}\right), e\left(q_{1}, p_{1}\right), e\left(p_{1}, q\right)$ and inserts the edge $e(p, q)$ transforms $\left(\Gamma_{f}, f_{\mid}\right)$into $\left(\Gamma_{g}, g_{\mid}\right)$with cost $c(T)=\frac{1}{2}\left(f\left(q_{1}\right)-\right.$ $\left.f\left(p_{1}\right)\right)$. Hence $d\left(\left(\Gamma_{f}, f_{\mid}\right),\left(\Gamma_{g}, g_{\mid}\right)\right)=\frac{1}{2}\left(f\left(q_{1}\right)-f\left(p_{1}\right)\right)$. 


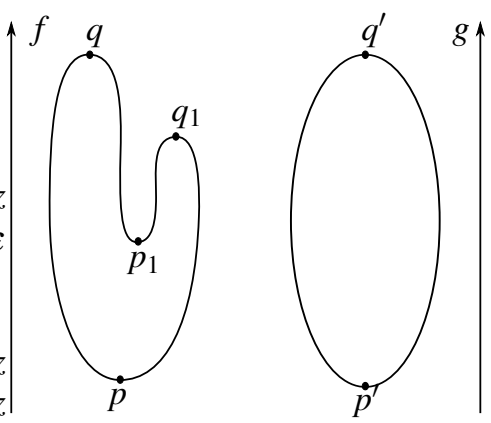

FIGURE 4. The functions $f, g \in \mathscr{F}^{0}$ considered in Example 1. In this case $d\left(\left(\Gamma_{f}, f_{\mid}\right),\left(\Gamma_{g}, g_{\mid}\right)\right)=\inf _{\tau \in \mathscr{H}\left(S^{1}\right)}\|f-g \circ \tau\|_{C^{0}}=\frac{1}{2}\left(f\left(q_{1}\right)-f\left(p_{1}\right)\right)$.

Example 2. Let us consider now $f, g: S^{1} \rightarrow \mathbb{R}$, with $f, g \in \mathscr{F}^{0}$, illustrated in Figure 5 . Let $f\left(q_{1}\right)-f\left(p_{1}\right)=f\left(q_{2}\right)-f\left(p_{2}\right)=a$. Then, clearly, $\inf _{\tau \in \mathscr{H}\left(S^{1}\right)}\|f-g \circ \tau\|_{C^{0}}=\frac{a}{2}$. Let us show that the editing distance between $\left(\Gamma_{f}, f_{\mid}\right)$and $\left(\Gamma_{g}, g_{\mid}\right)$is $\frac{a}{2}$, too. For every $0<\varepsilon<\frac{a}{2}$,
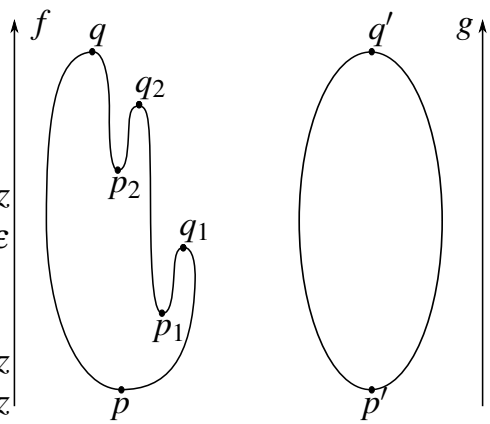

FIGURE 5. The functions $f, g \in \mathscr{F}^{0}$ considered in Example 2. Even in this case $d\left(\left(\Gamma_{f}, f_{\mid}\right),\left(\Gamma_{g}, g_{\mid}\right)\right)=\inf _{\tau \in \mathscr{H}\left(S^{1}\right)}\|f-g \circ \tau\|_{C^{0}}=\frac{1}{2}\left(f\left(q_{1}\right)-f\left(p_{1}\right)\right)$.

we can apply to $\left(\Gamma_{f}, f_{\mid}\right)$a deformation of type $(\mathrm{R})$, that relabels $p_{1}, p_{2}, q_{1}, q_{2}$ in such a way that $f\left(p_{i}\right)$ is increased of $\frac{a}{2}-\varepsilon$, and $f\left(q_{i}\right)$ is decreased of $\frac{a}{2}-\varepsilon$ for $i=1,2$, composed with two deformations of type (D) that delete $p_{i}$ with $q_{i}, i=1,2$. Thus, since the total cost is equal to $\frac{a}{2}-\varepsilon+2 \varepsilon$, by the arbitrariness of $\varepsilon$, it holds that $d\left(\left(\Gamma_{f}, f_{\mid}\right),\left(\Gamma_{g}, g_{\mid}\right)\right) \leq \frac{a}{2}$. Applying Theorem 3.1, we deduce that $d\left(\left(\Gamma_{f}, f_{\mid}\right),\left(\Gamma_{g}, g_{\mid}\right)\right)=\frac{a}{2}$.

\section{STABiLity}

This section is devoted to proving that Reeb graphs of closed curves are stable under arbitrary function perturbations. More precisely, it will be shown that arbitrary changes in simple Morse functions imply smaller changes in the editing distance between Reeb graphs.

4.1. Preliminaries. Let us endow $\mathscr{F}$ with the $C^{\infty}$ topology, and consider the strata $\mathscr{F}^{0}$ and $\mathscr{F}^{1}$ of the natural stratification of $\mathscr{F}$, as presented by Cerf in [2] (see also [16]).

- The stratum $\mathscr{F}^{0}$ is the set of simple Morse functions. 
- The stratum $\mathscr{F}^{1}$ is the disjoint union of two sets $\mathscr{F}_{\alpha}^{1}$ and $\mathscr{F}_{\beta}^{1}$ open in $\mathscr{F}^{1}$, where

- $\mathscr{F}_{\alpha}^{1}$ is the set of functions whose critical levels contain exactly one critical point, and the critical points are all non-degenerate, except exactly one. In a neighborhood of such a point, say $p$, a local coordinate system $x$ can be chosen such that $f=f(p)+x^{3}$.

- $\mathscr{F}_{\beta}^{1}$ is the set of Morse functions whose critical levels contain at most one critical point, except for one level containing exactly two critical points.

$\mathscr{F}^{0}$ is open and dense in the space $\mathscr{F}$ endowed with the $C^{r}$ topology, $2 \leq r \leq \infty$ (cf. [10, chap. 6, Thm. 1.2]). Therefore, any function of $\mathscr{F}^{1}$ can be turned into a simple Morse function by arbitrarily small perturbations. Degenerate critical points can be split into non-degenerate singularities, with different critical values (Figure $6(a)$ ). Moreover, when more than one critical point occur at the same level, they can be moved to close but different levels (Figure $6(b)$ ).

It is well-known that two simple Morse functions are topologically equivalent if and only if they belong to the same arcwise connected component (or co-cellule) of $\mathscr{F}^{0}[2$, p. 25].

$\mathscr{F}^{1}$ is a sub-manifold of co-dimension 1 of $\mathscr{F}^{0} \cup \mathscr{F}^{1}$, and the complement of $\mathscr{F}^{0} \cup \mathscr{F}^{1}$ in $\mathscr{F}$ is of co-dimension greater than 1 . Consequently, given two functions $f, g \in \mathscr{F}^{0}$, we can always find $\widehat{f}, \widehat{g} \in \mathscr{F}^{0}$ arbitrarily near to $f, g$, respectively, for which

- $\widehat{f}, \widehat{g}$ are topologically equivalent to $f, g$, respectively, and the path $h(\lambda)=(1-\lambda) \widehat{f}+\lambda \widehat{g}$, with $\lambda \in[0,1]$, is such that

- $h(\lambda)$ belongs to $\mathscr{F}^{0} \cup \mathscr{F}^{1}$ for every $\lambda \in[0,1]$;

- $h(\lambda)$ is transversal to $\mathscr{F}^{1}$.

As a consequence, $h(\lambda)$ belongs to $\mathscr{F}^{1}$ for at most a finite collection of values $\lambda$, and does not traverse strata of co-dimension greater than 1 (see, e.g., [9]).

4.2. Local Stability. We now prove the local stability of labeled Reeb graphs of closed curves. More precisely, we prove that each simple Morse function $f$ has a neighborhood consisting of simple Morse functions $g$ such that the editing distance between the labeled Reeb graphs of $f$ and $g$ is never greater that the $C^{0}$-norm of $f-g$.

We first need some lemmas.

Lemma 4.1. Let $f \in \mathscr{F}^{0}$ and let $c$ be a critical value of $f$. Then there exists a real number $\delta(f, c)>0$ such that each $g \in \mathscr{F}^{0}$ verifying $\|f-g\|_{C^{0}} \leq \delta(f, c)$, admits at least one critical value $c^{\prime}$ for which $\left|c-c^{\prime}\right| \leq\|f-g\|_{C^{0}}$.

The proof of the above result will be given in Appendix A for manifolds of arbitrary dimension.

Lemma 4.2. Let $f \in \mathscr{F}^{0}$. Then there exists a positive real number $\delta(f)$ such that, for every $g \in \mathscr{F}^{0}$, with $\|f-g\|_{C^{2}} \leq \delta(f)$, an edge and vertices-order preserving bijection $\Phi: V\left(\Gamma_{f}\right) \rightarrow V\left(\Gamma_{g}\right)$ exists for which $\max _{v \in V\left(\Gamma_{f}\right)}\left|f_{\mid}(v)-g_{\mid}(\Phi(v))\right| \leq\|f-g\|_{C^{0}}$.

Proof. Let $p_{1}, \ldots, p_{n}$ be the critical points of $f$, and $c_{1}, \ldots, c_{n}$ the respective critical values, with $c_{i}<c_{i+1}$ for $i=1 \ldots, n-1$. Since $\mathscr{F}^{0}$ is open in $\mathscr{F}$, endowed with the $C^{2}$ topology, there always exists a sufficiently small $\delta(f)>0$, such that the closed ball with center $f$ and radius $\delta(f), \overline{B_{2}(f, \delta(f))}$, is contained in $\mathscr{F}^{0}$. Moreover, $\delta(f)$ can be chosen so small that, for every $i=1, \ldots, n-1$, the intervals $\left[c_{i}-\delta(f), c_{i}+\delta(f)\right]$ and $\left[c_{i+1}-\delta(f), c_{i+1}+\delta(f)\right]$ are disjoint and $\delta(f)<\min _{i=1, \ldots, n} \delta\left(f, c_{i}\right)$, with $\delta\left(f, c_{i}\right)$ as in Lemma 4.1. 

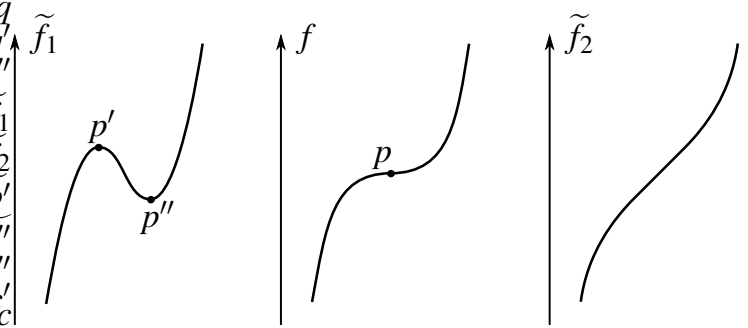

(a)

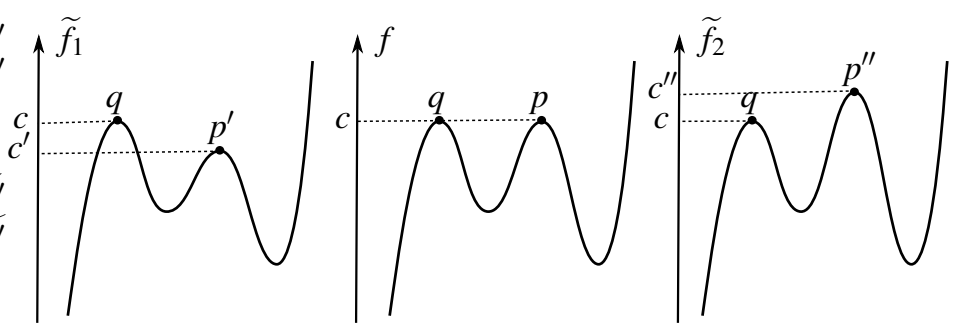

(b)

FIGURE 6. (a) A function $f \in \mathscr{F}_{\alpha}^{1}$ admitting one degenerate critical point $p$ (center) can be perturbed into a simple Morse function $\widetilde{f}_{1}$ with two non-degenerate critical points $p^{\prime}, p^{\prime \prime}$ (left), or into a simple Morse function $\widetilde{f}_{2}$ without critical points around $p$ (right); (b) a function $f \in \mathscr{F}_{\beta}^{1}$ (center) can be turned into two simple Morse functions $\widetilde{f}_{1}, \widetilde{f}_{2}$, that are not topologically equivalent (left-right).

Fixed such a $\delta(f)$, for every $g \in \mathscr{F}^{0}$ such that $\|f-g\|_{C^{2}} \leq \delta(f), f$ and $g$ belong to the same arcwise connected component of $\mathscr{F}^{0}$ endowed with the $C^{\infty}$ topology, and, therefore, are topologically equivalent functions. Consequently, there exists an edge and verticesorder preserving bijection $\Phi: V\left(\Gamma_{f}\right) \rightarrow V\left(\Gamma_{g}\right)$ (see Section 1).

Let us prove that $\Phi$ is such that $\max _{v \in V\left(\Gamma_{f}\right)}\left|f_{\mid}(v)-g_{\mid}(\Phi(v))\right| \leq\|f-g\|_{C^{0}}$. Since $f$ and $g$ are topologically equivalent, it follows that $g$ has exactly $n$ critical points, $p_{1}^{\prime}, \ldots, p_{n}^{\prime}$. Let $c_{1}^{\prime}=g\left(p_{1}^{\prime}\right), \ldots, c_{n}^{\prime}=g\left(p_{n}^{\prime}\right)$. We can assume $c_{i}^{\prime}<c_{i+1}^{\prime}$, for $i=1, \ldots, n-1$. The assumption $\|f-g\|_{C^{2}} \leq \delta(f)$ implies that $\|f-g\|_{C^{0}} \leq \delta(f)$. Therefore, recalling that $\delta(f)<\min _{i=1, \ldots, n} \delta\left(f, c_{i}\right)$, by Lemma 4.1, for every critical value $c_{i}$ of $f$, there exists at least one critical value $c_{i}^{\prime \prime}$ of $g$ of the same index of $c_{i}$ with $\left|c_{i}-c_{i}^{\prime \prime}\right| \leq\|f-g\|_{C^{0}}$. Moreover, since $\left[c_{i}-\delta(f), c_{i}+\delta(f)\right] \cap\left[c_{i+1}-\delta(f), c_{i+1}+\delta(f)\right]=\emptyset$ for every $i=1, \ldots, n-1$, and $\|f-g\|_{C^{0}} \leq \delta(f)$, it follows that $c_{i}^{\prime \prime} \in\left[c_{i}-\delta(f), c_{i}+\delta(f)\right]$ for every $i=1, \ldots, n$. Hence, since $\Phi$ preserves the order of the vertices, necessarily $\Phi\left(p_{i}\right)=p_{i}^{\prime}$ and $c_{i}^{\prime \prime}=c_{i}^{\prime}$, yielding that $\max _{v \in V\left(\Gamma_{f}\right)}\left|f_{\mid}(v)-g_{\mid}(\Phi(v))\right|=\max _{p_{i} \in K(f)}\left|f_{\mid}\left(p_{i}\right)-g_{\mid}\left(\Phi\left(p_{i}\right)\right)\right|=\max _{1 \leq i \leq n}\left|c_{i}-c_{i}^{\prime \prime}\right| \leq\|f-g\|_{C^{0}}$.

Theorem 4.3 (Local stability). Let $f \in \mathscr{F}^{0}$. Then there exists a positive real number $\delta(f)$ such that, for every $g \in \mathscr{F}^{0}$ with $\|f-g\|_{C^{2}} \leq \delta(f)$, it holds that

$$
d\left(\left(\Gamma_{f}, f_{\mid}\right),\left(\Gamma_{g}, g_{\mid}\right)\right) \leq\|f-g\|_{C^{0}} .
$$

Proof. By Lemma 4.2, an edge and vertices-order preserving bijection $\Phi: V\left(\Gamma_{f}\right) \rightarrow V\left(\Gamma_{g}\right)$ exists for which $\max _{v \in V\left(\Gamma_{f}\right)}\left|f_{\mid}(v)-g_{\mid}(\Phi(v))\right| \leq\|f-g\|_{C^{0}}$. Necessarily $\Phi$ takes minima into 
minima and maxima into maxima. Therefore, $\left(\Gamma_{f}, g_{\mid} \circ \Phi\right)=T\left(\Gamma_{f}, f_{\mid}\right)$, with $T$ an elementary deformation of type (R), relabeling vertices of $V\left(\Gamma_{f}\right)$, having $\operatorname{cost} c(T)=\max _{v \in V\left(\Gamma_{f}\right)} \mid f_{\mid}(v)-$ $g_{\mid}(\Phi(v)) \mid \leq\|f-g\|_{C^{0}}$. Moreover, let us observe that $\left(\Gamma_{f}, g_{\mid} \circ \Phi\right)$ is isomorphic to $\left(\Gamma_{g}, g_{\mid}\right)$ as labeled Reeb graph (see Definition 1.2). Thus, $d\left(\left(\Gamma_{f}, f_{\mid}\right),\left(\Gamma_{g}, g_{\mid}\right)\right)=d\left(\left(\Gamma_{f}, f_{\mid}\right),\left(\Gamma_{f}, g_{\mid} \circ\right.\right.$ $\Phi))=\inf _{T \in \mathscr{T}\left(\left(\Gamma_{f}, f_{\mid}\right),\left(\Gamma_{g}, g_{\mid}\right)\right)} c(T) \leq\|f-g\|_{C^{0}}$.

4.3. Global Stability. To prove the global stability of Reeb graphs, we proceed by steps: the following Proposition 4.4 shows such a stability property when the functions defined on $S^{1}$ belong to the same arcwise connected component of $\mathscr{F}^{0}$; Proposition 4.5 proves the same result in the case that the linear convex combination of two simple Morse functions traverses the stratum $\mathscr{F}^{1}$ at most in one point; Theorem 4.6 extends the result to two arbitrary functions in $\mathscr{F}^{0}$.

Proposition 4.4. Let $f, g \in \mathscr{F}^{0}$ and let $u s$ consider the path $h:[0,1] \rightarrow \mathscr{F}$ defined by $h(\lambda)=(1-\lambda) f+\lambda g$. If $h(\lambda) \in \mathscr{F}^{0}$ for every $\lambda \in[0,1]$, then $d\left(\left(\Gamma_{f}, f_{\mid}\right),\left(\Gamma_{g}, g_{\mid}\right)\right) \leq$ $\|f-g\|_{C^{0}}$.

Proof. Let $\delta(h(\lambda))>0$ be the fixed real number playing the same role of $\delta(f)$ in Theorem 4.3, after replacing $f$ by $h(\lambda)$. For conciseness, let us denote it by $\delta(\lambda)$, and $\|f-g\|_{C^{2}}$ by $a$. If $a=0$, then the claim trivially follows. If $a>0$, let $C$ be the open covering of $[0,1]$ constituted of open intervals $I_{\lambda}=\left(\lambda-\frac{\delta(\lambda)}{2 a}, \lambda+\frac{\delta(\lambda)}{2 a}\right)$. Let $C^{\prime}$ be a finite minimal (i.e. such that, for every $i, I_{\lambda_{i}} \nsubseteq \bigcup_{j \neq i} I_{\lambda_{j}}$ ) sub-covering of $C$, with $\lambda_{1}<\lambda_{2}<\ldots<\lambda_{n}$ the middle points of its intervals. Since $C^{\prime}$ is minimal, for every $i \in\{1, \ldots, n-1\}, I_{\lambda_{i}} \cap I_{\lambda_{i+1}}$ is non-empty. This implies that

$$
\lambda_{i+1}-\lambda_{i}<\frac{\delta\left(\lambda_{i}\right)}{2 a}+\frac{\delta\left(\lambda_{i+1}\right)}{2 a} \leq \frac{\max \left\{\delta\left(\lambda_{i}\right), \delta\left(\lambda_{i+1}\right)\right\}}{a} .
$$

Moreover, by the definition of $h$ and the linearity of derivatives, it can be deduced that

$$
\left\|h\left(\lambda_{i+1}\right)-h\left(\lambda_{i}\right)\right\|_{C^{2}}=\left(\lambda_{i+1}-\lambda_{i}\right) \cdot\|f-g\|_{C^{2}} .
$$

Now, substituting (4.1) in (4.2), we obtain

$$
\left\|h\left(\lambda_{i+1}\right)-h\left(\lambda_{i}\right)\right\|_{C^{2}}<\frac{\max \left\{\delta\left(\lambda_{i}\right), \delta\left(\lambda_{i+1}\right)\right\}}{a} \cdot\|f-g\|_{C^{2}}=\max \left\{\delta\left(\lambda_{i}\right), \delta\left(\lambda_{i+1}\right)\right\} .
$$

Let us consider the labeled Reeb graphs $\left(\Gamma_{h\left(\lambda_{i}\right)}, h\left(\lambda_{i}\right)_{\mid}\right)$with $i=1, \ldots, n$.

Let $i \in\{1, \ldots, n-1\}$. If $\max \left\{\delta\left(\lambda_{i}\right), \delta\left(\lambda_{i+1}\right)\right\}=\delta\left(\lambda_{i}\right)$, then using Theorem 4.3, with $f$ replaced by $h\left(\lambda_{i}\right)$ and $g$ by $h\left(\lambda_{i+1}\right)$, it holds that

$$
d\left(\left(\Gamma_{h\left(\lambda_{i}\right)}, h\left(\lambda_{i}\right)_{\mid}\right),\left(\Gamma_{h\left(\lambda_{i+1}\right)}, h\left(\lambda_{i+1}\right)_{\mid}\right)\right) \leq\left\|h\left(\lambda_{i+1}\right)-h\left(\lambda_{i}\right)\right\|_{C^{0}} .
$$

The same inequality holds when $\max \left\{\delta\left(\lambda_{i}\right), \delta\left(\lambda_{i+1}\right)\right\}=\delta\left(\lambda_{i+1}\right)$, as can be analogously checked.

Now, setting $\lambda_{0}=0, \lambda_{n+1}=1$, it can be verified that (4.3) also holds for $i=0, n$. Consequently, since $\Gamma_{f}=\Gamma_{h\left(\lambda_{0}\right)}$, and $\Gamma_{g}=\Gamma_{h\left(\lambda_{n+1}\right)}$, we have

$$
\begin{aligned}
d\left(\left(\Gamma_{f}, f_{\mid}\right),\left(\Gamma_{g}, g_{\mid}\right)\right) & \leq \sum_{i=0}^{n} d\left(\left(\Gamma_{h\left(\lambda_{i}\right)}, h\left(\lambda_{i}\right)_{\mid}\right),\left(\Gamma_{h\left(\lambda_{i+1}\right)}, h\left(\lambda_{i+1}\right)_{\mid}\right)\right) \leq \sum_{i=0}^{n}\left\|h\left(\lambda_{i+1}\right)-h\left(\lambda_{i}\right)\right\|_{C^{0}} \\
& =\sum_{i=0}^{n}\left(\lambda_{i+1}-\lambda_{i}\right) \cdot\|f-g\|_{C^{0}}=\|f-g\|_{C^{0}}
\end{aligned}
$$


where the first inequality is due to the triangular inequality, the second one to (4.3), the first equality holds because of (4.2), the second one because $\sum_{i=0}^{n}\left(\lambda_{i+1}-\lambda_{i}\right)=1$.

Proposition 4.5. Let $f, g \in \mathscr{F}^{0}$ and let us consider the path $h:[0,1] \rightarrow \mathscr{F}$ defined by $h(\lambda)=(1-\lambda) f+\lambda g$. If $h(\lambda) \in \mathscr{F}^{0}$ for every $\lambda \in[0,1] \backslash\{\bar{\lambda}\}$, with $0<\bar{\lambda}<1$, and $h$ transversely intersects $\mathscr{F}^{1}$ at $\bar{\lambda}$, then $d\left(\left(\Gamma_{f}, f_{\mid}\right),\left(\Gamma_{g}, g_{\mid}\right)\right) \leq\|f-g\|_{C^{0}}$.

Proof. We begin proving the following claim.

Claim. For every $\delta>0$ there exist two real numbers $\lambda^{\prime}, \lambda^{\prime \prime} \in[0,1]$, with $\lambda^{\prime}<\bar{\lambda}<\lambda^{\prime \prime}$, such that $d\left(\left(\Gamma_{h\left(\lambda^{\prime}\right)}, h\left(\lambda^{\prime}\right)_{\mid}\right),\left(\Gamma_{h\left(\lambda^{\prime \prime}\right)}, h\left(\lambda^{\prime \prime}\right)_{\mid}\right)\right) \leq \delta$.

To prove this claim, let us first assume that $h(\bar{\lambda})$ belongs to $\mathscr{F}_{\alpha}^{1}$. To simplify the notation, we denote $h(\bar{\lambda})$ simply by $\bar{h}$. Let $\bar{p}$ be the sole degenerate critical point for $\bar{h}$. It is well known that there exists a suitable local coordinate system $x$ around $\bar{p}$ in which the canonical expression of $\bar{h}$ is $\bar{h}=\bar{h}(\bar{p})+x^{3}$ (see Figure $6(a)$ with $\bar{h}$ replaced by $f$ ).

Let us take a smooth function $\omega: S^{1} \rightarrow \mathbb{R}$ whose support is contained in the coordinate chart around $\bar{p}$ in which $\bar{h}=\bar{h}(\bar{p})+x^{3}$; moreover, let us assume that $\omega$ is equal to 1 in a neighborhood of $\bar{p}$, and decreases moving from $\bar{p}$. Let us consider the family of smooth functions $\bar{h}_{t}$ obtained by locally modifying $\bar{h}$ near $\bar{p}$ as follows: $\bar{h}_{t}=\bar{h}+t \cdot \omega \cdot x$. There exists $\bar{t}>0$ sufficiently small such that $(i)$ for $0<t \leq \bar{t}, \bar{h}_{t}$ has no critical points in the support of $\omega$ and is equal to $\bar{h}$ everywhere else (see Figure $6(a)$ with $\bar{h}_{t}$ replaced by $\widetilde{f}_{2}$ ), and $(i i)$ for $-\bar{t} \leq t<0, \bar{h}_{t}$ has exactly two critical points in the support of $\omega$ whose values difference tends to vanish as $t$ tends to 0 , and $\bar{h}_{t}$ is equal to $\bar{h}$ everywhere else (see [2] and Figure $6(a)$ with $\bar{h}_{t}$ replaced by $\left.\widetilde{f}_{1}\right)$.

Since $\bar{h}_{t}$ is a universal deformation of $\bar{h}=h(\bar{\lambda})$, and $h$ intersect $\mathscr{F}^{1}$ transversely at $\bar{\lambda}$, either the maps $h(\lambda)$ with $\lambda<\bar{\lambda}$ are topologically equivalent to $h_{t}$ with $t>0$ or to $h_{t}$ with $t<0$ (cf. [2, 12, 16]). Analogously for the maps $h(\lambda)$ with $\lambda>\bar{\lambda}$. Let us assume that $h(\lambda)$ is topologically equivalent to $h_{t}$ with $t<0$ when $\lambda<\bar{\lambda}$, while $h(\lambda)$ is topologically equivalent to $h_{t}$ with $t>0$ when $\lambda>\bar{\lambda}$. Hence, for every $\delta>0$, there exist $\lambda^{\prime}$, with $0 \leq \lambda^{\prime}<\bar{\lambda}$, and $\lambda^{\prime \prime}$, with $\bar{\lambda}<\lambda^{\prime \prime} \leq 1$, such that $h\left(\lambda^{\prime}\right)$ and $h\left(\lambda^{\prime \prime}\right)$ have the same critical points, with the same values, except for two critical points of $h\left(\lambda^{\prime}\right)$, whose values difference is smaller than $\delta$, that are non-critical for $h\left(\lambda^{\prime \prime}\right)$. Therefore, $\left(\Gamma_{h\left(\lambda^{\prime}\right)}, h\left(\lambda^{\prime}\right)_{\mid}\right)$can be transformed into $\left(\Gamma_{h\left(\lambda^{\prime \prime}\right)}, h\left(\lambda^{\prime \prime}\right) \mid\right)$ by an elementary deformation of type (D) whose cost is not greater than $\delta$. In the case when $h(\lambda)$ is topologically equivalent to $h_{t}$ with $t>0$ when $\lambda<\bar{\lambda}$, while $h(\lambda)$ is topologically equivalent to $h_{t}$ with $t<0$ when $\lambda>\bar{\lambda}$, the claim can be proved similarly, applying an elementary deformation of type (B).

Let us now prove the claim when $\bar{h}=h(\bar{\lambda})$ belongs to $\mathscr{F}_{\beta}^{1}$. Let us denote by $\bar{p}$ and $\bar{q}$ the critical points of $\bar{h}$ such that $\bar{h}(\bar{p})=\bar{h}(\bar{q})$. Since $\bar{p}$ is non-degenerate there exists a suitable local coordinate system $x$ around $\bar{p}$ in which the canonical expression of $\bar{h}$ is $\bar{h}=\bar{h}(\bar{p})+x^{2}$ (see Figure $6(b)$ with $\bar{h}$ replaced by $f$ ). Let us take $\omega$ as before, whose support is contained in such a coordinate chart. Let us locally modify $\bar{h}$ near $\bar{p}$ as follows: $\bar{h}_{t}=\bar{h}+t \cdot \omega$. There exists $\bar{t}>0$ sufficiently small such that for $|t| \leq \bar{t}, \bar{h}_{t}$ has exactly the same critical points as $\bar{h}$. As for critical values, they are the same as well, apart from the value taken at $\bar{p}$ : $\bar{h}_{t}(\bar{p})<\bar{h}(\bar{p})$, for $-\bar{t} \leq t<0$ (see Figure $6(b)$ with $\bar{h}_{t}$ replaced by $\widetilde{f}_{1}$ ), while $\bar{h}_{t}(\bar{p})>\bar{h}(\bar{p})$, for $0<t \leq \bar{t}$ (see Figure $6(b)$ with $\bar{h}_{t}$ replaced by $\widetilde{f}_{2}$ ), and $\bar{h}_{t}(\bar{p})$ tends to $\bar{h}(\bar{p})$ as $t$ tends to 0 (cf. [2]). Since $\bar{h}_{t}$ is a universal deformation of $\bar{h}=h(\bar{\lambda})$, and $h$ intersect $\mathscr{F}^{1}$ transversely at $\bar{\lambda}$, we deduce that for every $\delta>0$ there exist $\lambda^{\prime}$, with 
$0 \leq \lambda^{\prime}<\bar{\lambda}$ and $\lambda^{\prime \prime}$, with $\bar{\lambda}<\lambda^{\prime \prime} \leq 1$, such that $\left(\Gamma_{h\left(\lambda^{\prime}\right)}, h\left(\lambda^{\prime}\right)_{\mid}\right)$can be transformed into $\left(\Gamma_{h\left(\lambda^{\prime \prime}\right)}, h\left(\lambda^{\prime \prime}\right)\right)$ by an elementary deformation of type $(\mathrm{R})$ whose cost is not greater than $\delta$. Therefore the initial claim is proved.

Let us now estimate $d\left(\left(\Gamma_{f}, f_{1}\right),\left(\Gamma_{g}, g_{1}\right)\right)$. By the claim, for every $\delta>0$, there exist $0<\lambda^{\prime}<\lambda^{\prime \prime}<1$ such that, applying the triangular inequality,

$$
\begin{aligned}
d\left(\left(\Gamma_{f}, f_{\mid}\right),\left(\Gamma_{g}, g_{\mid}\right)\right) \leq & d\left(\left(\Gamma_{f}, f_{\mid}\right),\left(\Gamma_{h\left(\lambda^{\prime}\right)}, h\left(\lambda^{\prime}\right)_{\mid}\right)\right)+d\left(\left(\Gamma_{h\left(\lambda^{\prime}\right)}, h\left(\lambda^{\prime}\right)_{\mid}\right),\left(\Gamma_{h\left(\lambda^{\prime \prime}\right)}, h\left(\lambda^{\prime \prime}\right)_{\mid}\right)\right) \\
& +d\left(\left(\Gamma_{h\left(\lambda^{\prime \prime}\right)}, h\left(\lambda^{\prime \prime}\right)_{\mid}\right),\left(\Gamma_{g}, g_{\mid}\right)\right) \\
\leq & d\left(\left(\Gamma_{f}, f_{\mid}\right),\left(\Gamma_{h\left(\lambda^{\prime}\right)}, h\left(\lambda^{\prime}\right)_{\mid}\right)\right)+d\left(\left(\Gamma_{h\left(\lambda^{\prime \prime}\right)}, h\left(\lambda^{\prime \prime}\right)_{\mid}\right),\left(\Gamma_{g}, g_{\mid}\right)\right)+\delta .
\end{aligned}
$$

By Proposition 4.4,

$$
d\left(\left(\Gamma_{f}, f_{\mid}\right),\left(\Gamma_{h\left(\lambda^{\prime}\right)}, h\left(\lambda^{\prime}\right)_{\mid}\right)\right) \leq\left\|f-h\left(\lambda^{\prime}\right)\right\|_{C^{0}}=\lambda^{\prime} \cdot\|f-g\|_{C^{0}},
$$

and

$$
d\left(\left(\Gamma_{h\left(\lambda^{\prime \prime}\right)}, h\left(\lambda^{\prime \prime}\right)_{\mid}\right),\left(\Gamma_{g}, g_{\mid}\right)\right) \leq\left\|h\left(\lambda^{\prime \prime}\right)-g\right\|_{C^{0}}=\left(1-\lambda^{\prime \prime}\right) \cdot\|f-g\|_{C^{0}} .
$$

Hence, $d\left(\left(\Gamma_{f}, f_{\mid}\right),\left(\Gamma_{g}, g_{\mid}\right)\right) \leq\|f-g\|_{C^{0}}+\delta$, yielding the conclusion by the arbitrariness of $\delta$.

Theorem 4.6 (Global stability). Let $f, g \in \mathscr{F}^{0}$. Then

$$
d\left(\left(\Gamma_{f}, f_{\mid}\right),\left(\Gamma_{g}, g_{\mid}\right)\right) \leq\|f-g\|_{C^{0}} .
$$

Proof. For every sufficiently small $\delta>0$ such that the balls with center $f$ and $g$ and radius $\delta$ with respect to the $C^{2}$-norm, $B_{2}(f, \delta), B_{2}(g, \delta)$, are contained in $\mathscr{F}^{0}$, there exist $\widehat{f} \in$ $B_{2}(f, \delta)$ and $\widehat{g} \in B_{2}(g, \delta)$ such that the path $h:[0,1] \rightarrow \mathscr{F}$, with $h(\lambda)=(1-\lambda) \widehat{f}+\lambda \widehat{g}$, belongs to $\mathscr{F}^{0}$ for every $\lambda \in[0,1]$, except for at most a finite number $n$ of values $0<\mu_{1}<$ $\mu_{2}<\ldots<\mu_{n}<1$ at which $h$ transversely intersects $\mathscr{F}^{1}$. If $n=0$ ( $n=1$, respectively), then the claim immediately follows from Proposition 4.4 (Proposition 4.5, respectively). If $n>1$, let $0<\lambda_{1}<\lambda_{2}<\ldots<\lambda_{2 n-1}<1$, with $\lambda_{2 i-1}=\mu_{i}$ for $i=1, \ldots, n$. Then $h\left(\lambda_{2 i-1}\right) \in$ $\mathscr{F}^{1}$ for $i=1, \ldots, n, h\left(\lambda_{2 i}\right) \in \mathscr{F}^{0}$ for $i=1, \ldots, n-1$. Set $\lambda_{0}=0$ so that $\widehat{f}=h\left(\lambda_{0}\right)$, and $\lambda_{2 n}=1$ so that $\widehat{g}=h\left(\lambda_{2 n}\right)$ (a schematization of this path is illustrated in Figure 7). Then, by Proposition 4.5, we have

$$
d\left(\left(\Gamma_{h\left(\lambda_{2 i}\right)}, h\left(\lambda_{2 i}\right)_{\mid}\right),\left(\Gamma_{h\left(\lambda_{2 i+2}\right)}, h\left(\lambda_{2 i+2}\right)_{\mid}\right)\right) \leq\left\|h\left(\lambda_{2 i}\right)-h\left(\lambda_{2 i+2}\right)\right\|_{C^{0}}
$$

for every $i=0, \ldots, n-1$. Therefore

$$
\begin{aligned}
d\left(\left(\Gamma_{\widehat{f}}, \widehat{f}_{\mid}\right),\left(\Gamma_{\widehat{g}}, \widehat{g} \mid\right)\right) & \leq \sum_{i=0}^{n-1} d\left(\left(\Gamma_{h\left(\lambda_{2 i}\right)}, h\left(\lambda_{2 i}\right)_{\mid}\right),\left(\Gamma_{h\left(\lambda_{2 i+2}\right)}, h\left(\lambda_{2 i+2}\right)_{\mid}\right)\right) \\
& \leq \sum_{i=0}^{n-1}\left\|h\left(\lambda_{2 i}\right)-h\left(\lambda_{2 i+2}\right)\right\|_{C^{2}} \leq\|\widehat{f}-\widehat{g}\|_{C^{0}} .
\end{aligned}
$$

Then, recalling that $\widehat{f} \in B_{2}(f, \delta)$ means $\|\widehat{f}-f\|_{C^{2}} \leq \delta$, and $B_{2}(f, \delta) \subset \mathscr{F}^{0}$ implies that $(1-\lambda) f+\lambda \widehat{f} \in \mathscr{F}^{0}$ for every $\lambda \in[0,1]$, we can apply Proposition 4.4 to state that

$$
d\left(\left(\Gamma_{f}, f_{\mid}\right),\left(\Gamma_{\widehat{f}}, \widehat{f}_{\mid}\right)\right) \leq\|\widehat{f}-f\|_{C^{0}} \leq\|\widehat{f}-f\|_{C^{2}} \leq \delta .
$$

It is analogous for $g$ and $\widehat{g}$. Thus, from the triangular inequality, we have

$$
\begin{aligned}
d\left(\left(\Gamma_{f}, f_{\mid}\right),\left(\Gamma_{g}, g_{\mid}\right)\right) & \leq d\left(\left(\Gamma_{f}, f_{\mid}\right),\left(\Gamma_{\widehat{f}}, \widehat{f}_{\mid}\right)\right)+d\left(\left(\Gamma_{\widehat{f}}, \widehat{f}_{\mid}\right),\left(\Gamma_{\widehat{g}}, \widehat{g}_{\mid}\right)\right)+d\left(\left(\Gamma_{\widehat{g}}, \widehat{g}_{\mid}\right),\left(\Gamma_{g}, g_{\mid}\right)\right) \\
& \leq 2 \delta+\|\widehat{f}-\widehat{g}\|_{C^{0}} .
\end{aligned}
$$


Now, since by the triangular inequality, $\|\widehat{f}-\widehat{g}\|_{C^{0}} \leq\|\widehat{f}-f\|_{C^{0}}+\|f-g\|_{C^{0}}+\|g-\widehat{g}\|_{C^{0}}$, with $\|\widehat{f}-f\|_{C^{0}} \leq \delta$, and $\|g-\widehat{g}\|_{C^{0}} \leq \delta$, it follows that $d\left(\left(\Gamma_{f}, f_{\mid}\right),\left(\Gamma_{g}, g\right)\right) \leq 4 \delta+\|f-g\|_{C^{0}}$. Finally, because of the arbitrariness of $\delta$, we can let $\delta$ tend to zero and obtain the claim.

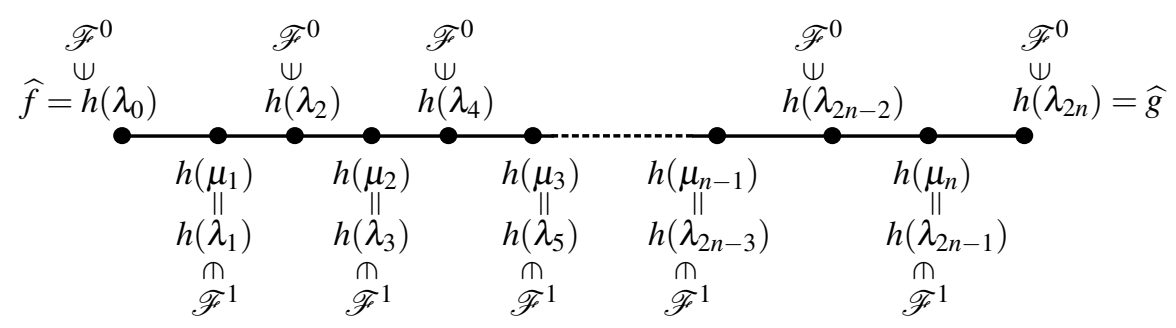

FIGURE 7. The linear path used in the proof of Theorem 4.6.

\section{RELATIONSHIPS WITH OTHER DISTANCES}

Corollary 5.1. For every $f, g \in \mathscr{F}^{0}, d\left(\left(\Gamma_{f}, f_{\mid}\right),\left(\Gamma_{g}, g_{\mid}\right)\right)$is equal to the natural pseudodistance between $f$ and $g$.

Proof. By Theorem 4.6, for every $\tau \in \mathscr{H}\left(S^{1}\right), d\left(\left(\Gamma_{f}, f_{\mid}\right),\left(\Gamma_{g \circ \tau}, g \circ \tau\right)\right) \leq\|f-g \circ \tau\|_{C^{0}}$. Moreover, for every $\tau \in \mathscr{H}\left(S^{1}\right), d\left(\left(\Gamma_{f}, f_{\mid}\right),\left(\Gamma_{g}, g_{l}\right)\right)=d\left(\left(\Gamma_{f}, f_{1}\right),\left(\Gamma_{g \circ \tau}, g \circ \tau_{\mid}\right)\right)$. Hence $d\left(\left(\Gamma_{f}, f_{\mid}\right),\left(\Gamma_{g}, g_{\mid}\right)\right) \leq \inf _{\tau \in \mathscr{H}\left(S^{1}\right)}\|f-g \circ \tau\|_{C^{0}}$. Recalling that, by Theorem 3.1, also the inequality $d\left(\left(\Gamma_{f}, f_{\mid}\right),\left(\Gamma_{g}, g_{\mid}\right)\right) \geq \inf _{\tau \in \mathscr{H}\left(S^{1}\right)}\|f-g \circ \tau\|_{C^{0}}$ holds, the claim follows.

Corollary 5.2. For every $f, g \in \mathscr{F}^{0}, d\left(\left(\Gamma_{f}, f_{\mid}\right),\left(\Gamma_{g}, g_{\mid}\right)\right)$is greater than or equal to the bottleneck distance between the persistence diagrams of $f$ and $g$.

Proof. The claim immediately follows from Corollary 5.1 and the fact that the bottleneck distance is a lower bound for the natural pseudo-distance (cf. [4]).

Remark 5.3. The editing distance between Reeb graphs is not equal to the bottleneck distance between the corresponding persistence diagrams.

To see this fact we exhibit in Figure 8 an example in which the editing distance between Reeb graphs is strictly greater than the bottleneck distance between the corresponding persistence diagrams. Indeed, the two curves have the same persistence diagrams for any homology degree but their natural pseudo-distance is non-zero since a homeomorphism $\tau: S^{1} \rightarrow S^{1}$ such that $f=g \circ \tau$ should take critical points of $f$ into critical points of $g$ preserving their values and adjacencies, which is clearly impossible.

\section{REFERENCES}

1. V. Arnold, Topological classification of Morse functions and generalisations of Hilbert's 16-th problem, Mathematical Physics, Analysis and Geometry 10 (2007), no. 3, 227-236.

2. J. Cerf, La stratification naturelle des espaces de fonctions différentiables réelles et le théorème de la pseudoisotopie., Inst. Hautes Études Sci. Publ. Math. (1970), no. 39, 5-173 (French).

3. A. Cerri and B. Di Fabio, On certain optimal homeomorphisms between closed curves, Tech. Rep. 2631, Università di Bologna, August 2010, http://amsacta.cib.unibo.it/2631/. 

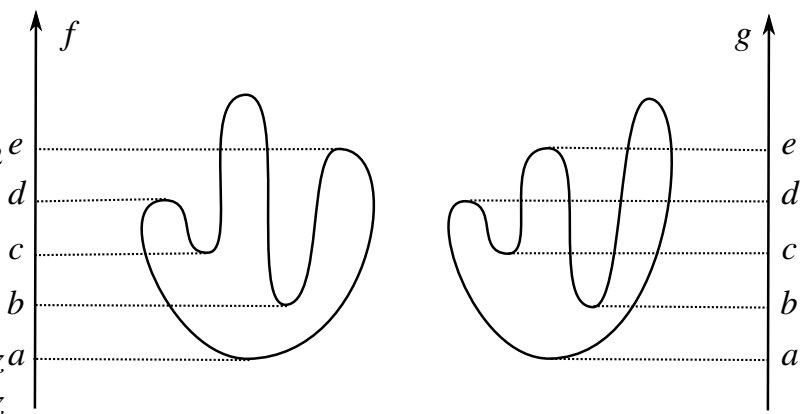

FIGURE 8. Two curves with the same persistence diagrams for any homology degree, whose natural pseudo-distance is non-zero.

4. A. Cerri, B. Di Fabio, M. Ferri, P. Frosini, and C. Landi, Betti numbers in multidimensional persistent homology are stable functions, Tech. Rep. 2923, Università di Bologna, November 2010, http://amsacta.cib.unibo.it/2923/.

5. D. Cohen-Steiner, H. Edelsbrunner, and J. Harer, Stability of persistence diagrams, Discrete Comput. Geom. 37 (2007), no. 1, 103-120.

6. P. Donatini and P. Frosini, Natural pseudodistances between closed manifolds, Forum Mathematicum 16 (2004), no. 5, 695-715.

7. __ Natural pseudodistances between closed surfaces, Journal of the European Mathematical Society 9 (2007), no. 2, 231-253.

8. __ Natural pseudo-distances between closed curves, Forum Mathematicum 21 (2009), no. 6, 981-999.

9. H. Edelsbrunner and J. Harer, Jacobi sets of multiple Morse functions, Foundations of Computational Mathematics (2002), 37-57.

10. M. Hirsch, Differential topology, Springer-Verlag, New York, 1976.

11. A. Marino and G. Prodi, Metodi perturbativi nella teoria di Morse, Boll. Un. Mat. Ital. (4) 11 (1975), no. 3, suppl., 1-32.

12. J. Martinet, Singularities of smooth functions and maps, London Mathematical Society Lecture Note Series, 58: Cambridge University Press. XIV, 1982.

13. J. Milnor, Morse theory, Based on lecture notes by M. Spivak and R. Wells. Annals of Mathematics Studies, no. 51, Princeton University Press, Princeton, N.J., 1963.

14. J. Palis and W. de Melo, Geometric theory of dynamical systems. An introduction., New York - Heidelberg Berlin: Springer-Verlag, 1982.

15. G. Reeb, Sur les points singuliers d'une forme de Pfaff complétement intégrable ou d'une fonction numérique, Comptes Rendus de L'Académie ses Sciences 222 (1946), 847-849.

16. F. Sergeraert, Un théorème de fonctions implicites sur certains espaces de Fréchet et quelques applications, Ann. Sci. École Norm. Sup. 5 (1972), 599-660 (French).

17. Y. Shinagawa and T. L. Kunii, Constructing a Reeb Graph automatically from cross sections, IEEE Computer Graphics and Applications 11 (1991), no. 6, 44-51.

18. Y. Shinagawa, T. L. Kunii, and Y.L. Kergosien, Surface coding based on Morse theory, IIEEE Computer Graphics and Applications 11 (1991), no. 5, 66-78.

19. Kuo-Chung Tai, The tree-to-tree correction problem, J. ACM 26 (1979), no. 3, 422-433.

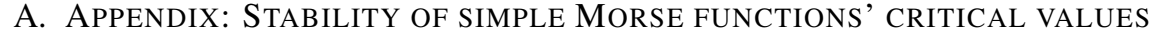

In this section we give a proof that critical values of simple Morse function are stable. We only use the homological properties of the lower level sets of a simple Morse function $f$ on a manifold $\mathscr{M}$, and its validity does not depend on the dimension of $\mathscr{M}$. Therefore, it will be given for any smooth compact manifold without boundary.

For every $C^{\infty}$ function $f: \mathscr{M} \rightarrow \mathbb{R}$, and for every $a \in \mathbb{R}$, let us denote by $f^{a}$ the lower level set $f^{-1}((-\infty, a])$. Let us recall the existing link between the topology of a pair of 
lower level sets $\left(f^{b}, f^{a}\right)$, with $a, b \in \mathbb{R}, a<b$, regular values of $f$, and the critical points of $f$ lying between $a$ and $b$ (cf. [13]):

(St. 1) If $f^{-1}([a, b])$ contains no critical points, then $f^{a}$ is a deformation retract of $f^{b}$, so that the inclusion map $f^{a} \rightarrow f^{b}$ is a homotopy equivalence.

(St. 2) If $f^{-1}([a, b])$ contains exactly one critical point of index $\bar{k}$, then, denoting by $G$ the homology coefficient group, it holds that

$$
H_{k}\left(f^{b}, f^{a}\right)= \begin{cases}G, & \text { if } k=\bar{k} \\ 0, & \text { otherwise. }\end{cases}
$$

In the remainder of this section we require $f$ to be a simple Morse function. Accordingly, it makes sense to use the terminology critical value of index $k$ to indicate a critical value that is the image of a critical point of index $k$.

Lemma A.1. Let $f: \mathscr{M} \rightarrow \mathbb{R}$ be a simple Morse function, and let $a, b \in \mathbb{R}, a<b$, be regular values of $f$. If there exists $\bar{k} \in \mathbb{Z}$ such that $H_{\bar{k}}\left(f^{b}, f^{a}\right) \neq 0$, then $[a, b]$ contains at least one critical value of index $\bar{k}$.

Proof. From (St. 1), the absence of critical values in $[a, b]$ implies that the homomorphism induced by inclusion $i_{k}: H_{k}\left(f^{a}\right) \rightarrow H_{k}\left(f^{b}\right)$ is an isomorphism for each $k \in \mathbb{Z}$. Consequently, by using the long exact sequence of the pair:

$$
\cdots \longrightarrow H_{k}\left(f^{a}\right) \stackrel{i_{k}}{\longrightarrow} H_{k}\left(f^{b}\right) \stackrel{j_{k}}{\longrightarrow} H_{k}\left(f^{b}, f^{a}\right) \stackrel{\partial_{k}}{\longrightarrow} H_{k-1}\left(f^{a}\right) \stackrel{i_{k-1}}{\longrightarrow} H_{k-1}\left(f^{b}\right) \longrightarrow \cdots,
$$

it is easily seen that, for every $k \in \mathbb{Z}$, the surjectivity of $i_{k}$ and the injectivity of $i_{k-1}$ imply the triviality of $H_{k}\left(f^{b}, f^{a}\right)$. This proves that if there exists $\bar{k} \in \mathbb{Z}$ such that $H_{\bar{k}}\left(f^{b}, f^{a}\right) \neq 0$, then $[a, b]$ contains at least one critical value of $f$. Moreover, as a consequence of (St. 2), the index of at least one of the critical values of $f$ contained in $[a, b]$ is exactly $\bar{k}$. Indeed, let $c_{1}, \ldots, c_{m}$ be the critical values of $f$ belonging to $[a, b]$, and let $s_{0}, \ldots, s_{m}$ be $m+1$ regular values such that $a=s_{0}<c_{1}<s_{1}<c_{2}<\ldots<s_{m-1}<c_{m}<s_{m}=b$. Since it holds that $\operatorname{rank} H_{\bar{k}}\left(f^{b}, f^{a}\right) \leq \sum_{i=1}^{m} \operatorname{rank} H_{\bar{k}}\left(f^{s_{i}}, f^{s_{i-1}}\right)$, and we are assuming $\operatorname{rank} H_{\bar{k}}\left(f^{b}, f^{a}\right) \geq 1$, there exists at least one index $i \in\{1, \ldots, m\}$ such that $H_{\bar{k}}\left(f^{s_{i}}, f^{s_{i-1}}\right) \neq 0$. Now, applying (St. 2) with $a$ replaced by $s_{i-1}$ and $b$ replaced by $s_{i}$, we deduce that $c_{i}$ is a critical value of $f$ of index $\bar{k}$.

The above statements (St. 1-2), Lemma A.1, together with the following lemma, that is a reformulation of Lemma 4.1 in [11], provide the tools for proving the stability of critical values under small function perturbations (Theorem A.3).

Lemma A.2. Let $X_{1}, X_{2}, X_{3}, X_{1}^{\prime}, X_{2}^{\prime}, X_{3}^{\prime}$ be topological spaces such that $X_{1} \subseteq X_{2} \subseteq X_{3} \subseteq$ $X_{1}^{\prime} \subseteq X_{2}^{\prime} \subseteq X_{3}^{\prime}$. Let $H_{k}\left(X_{3}, X_{1}\right)=0, H_{k}\left(X_{3}^{\prime}, X_{1}^{\prime}\right)=0$ for every $k \in \mathbb{Z}$. Then the homomorphism induced by inclusion $H_{k}\left(X_{1}^{\prime}, X_{1}\right) \rightarrow H_{k}\left(X_{2}^{\prime}, X_{2}\right)$ is injective for every $k \in \mathbb{Z}$.

Theorem A.3 (Stability of critical values). Let $f: \mathscr{M} \rightarrow \mathbb{R}$ be a simple Morse function and let $c$ be a critical value of index $\bar{k}$ of $f$. Then there exists a real number $\delta(f, c)>0$ such that each simple Morse function $g: \mathscr{M} \rightarrow \mathbb{R}$ verifying $\|f-g\|_{C^{0}} \leq \delta(f, c)$, admits at least one critical value $c^{\prime}$ of index $\bar{k}$ for which $\left|c-c^{\prime}\right| \leq\|f-g\|_{C^{0}}$.

Proof. Since $f$ is Morse, we can choose a real number $\delta(f, c)>0$ such that $[c-3$. $\delta(f, c), c+3 \cdot \delta(f, c)]$ does not contain any critical value of $f$ besides $c$. Let $g$ be a simple Morse function such that $\|f-g\|_{C^{0}} \leq \delta(f, c)$. If $g=f$, then the claim immediately follows. Let $g \neq f$ and denote $\|f-g\|_{C^{0}}$ by $\delta^{\prime}$. Then, for every $n \in \mathbb{N}$,

$$
f^{c-\delta^{\prime} \cdot \frac{2 n+1}{n}} \subseteq g^{c-\delta^{\prime} \cdot \frac{n+1}{n}} \subseteq f^{c-\delta^{\prime} / n} \subseteq f^{c+\delta^{\prime} / n} \subseteq g^{c+\delta^{\prime} \cdot \frac{n+1}{n}} \subseteq f^{c+\delta^{\prime} \cdot \frac{2 n+1}{n}} .
$$


Since $\left[c-\delta^{\prime} \cdot \frac{2 n+1}{n}, c-\delta^{\prime} / n\right]$ and $\left[c+\delta^{\prime} / n, c+\delta^{\prime} \cdot \frac{2 n+1}{n}\right]$ do not contain any critical value of $f$ for any $n \in \mathbb{N}$, both $H_{k}\left(f^{c-\delta^{\prime} / n}, f^{c-\delta^{\prime} \cdot \frac{2 n+1}{n}}\right)$ and $H_{k}\left(f^{c+\delta^{\prime} \cdot \frac{2 n+1}{n}}, f^{c+\delta^{\prime} / n}\right)$ are trivial for every $k \in \mathbb{Z}$, and $n \in \mathbb{N}$. Consequently, from Lemma A.2, the homomorphism induced by inclusion $H_{k}\left(f^{c+\delta^{\prime} / n}, f^{c-\delta^{\prime} \cdot \frac{2 n+1}{n}}\right) \rightarrow H_{k}\left(g^{c+\delta^{\prime} \cdot \frac{n+1}{n}}, g^{c-\delta^{\prime} \cdot \frac{n+1}{n}}\right)$ is injective for each $k \in \mathbb{Z}$, and $n \in \mathbb{N}$. Moreover, since, for every $n \in \mathbb{N},\left[c-\delta^{\prime} \cdot \frac{2 n+1}{n}, c+\delta^{\prime} / n\right]$ contains $c$, that is a critical value of index $\bar{k}$ of $f$, from (St. 2), it holds that $H_{\bar{k}}\left(f^{c+\delta^{\prime} / n}, f^{c-\delta^{\prime} \cdot \frac{2 n+1}{n}}\right) \neq 0$ for every $n \in \mathbb{N}$. This fact, together with the injectivity of the above map, implies that also $H_{\bar{k}}\left(g^{c+\delta^{\prime} \cdot \frac{n+1}{n}}, g^{c-\delta^{\prime} \cdot \frac{n+1}{n}}\right) \neq 0$ for every $n \in \mathbb{N}$. So, by Lemma A. 1 , for every $n \in \mathbb{N}$, there exists at least one critical value $c_{n}^{\prime}$ of index $\bar{k}$ of $g$ with $c_{n}^{\prime} \in\left(c-\delta^{\prime} \cdot \frac{n+1}{n}, c+\delta^{\prime} \cdot \frac{n+1}{n}\right)$. Now, by contradiction, let us assume that $\left[c-\delta^{\prime}, c+\delta^{\prime}\right]$ contains no critical values of index $\bar{k}$ of $g$. Then, since $g$ is Morse, there would exist a sufficiently small real number $\varepsilon>0$ such that $\left(c-\delta^{\prime}-\varepsilon, c+\delta^{\prime}+\varepsilon\right)$ does not contain critical values of index $\bar{k}$ of $g$ either, yielding a contradiction.

Dipartimento di Matematica, Università di Bologna, P.zZa di Porta S. Donato 5, I-40126 BOLOGNA, ITALIA

ARCES, UNIVERSiTÀ Di BOLOGNA, VIA TOFFANo 2/2, I-40135 BOLOGNA, ITALIA

E-mail address: difabio@dm. unibo.it

Dipartimento di SCIENZE E Metodi DELL'IngEgneria, Università di Modena E REgGio Emilia, Via Amendola 2, Pad. Morselli, I-42100 Reggio Emilia, Italia

ARCES, UNiversità Di Bologna, Via TOFFANo 2/2, I-40135 Bologna, ItALIA

E-mail address: clandi@unimore.it 